\title{
THEORY OF SITE-DISORDERED MAGNETS
}

\author{
Th.M. Nieuwenhuizen ${ }^{(1)}$ and C.N.A. van Duin ${ }^{(2)}$ \\ (1) University of Amsterdam, Van der Waals-Zeeman Instituut \\ Valckenierstraat 65-67, 1018 XE Amsterdam, The Netherlands \\ ${ }^{(2)}$ Institute Lorentz for Theoretical Physics, Leiden University \\ P.O.B. 9506, 2300 RA Leiden, The Netherlands
}

(September 3, 2018)

\begin{abstract}
In realistic spinglasses, such as CuMn, AuFe and EuSrS, magnetic atoms are located at random positions. Their couplings are determined by their relative positions. For such systems a field theory is formulated. In certain limits it reduces to the Hopfield model, the Sherrington-Kirkpatrick model, and the Viana-Bray model. The model has a percolation transition, while for RKKY couplings the "concentration scaling" $T_{g} \sim c$ occurs.

Within the Gaussian approximation the Ginzburg-Landau expansion is considered in the clusterglass phase, that is to say, for not too small concentrations. Near special points, the prefactor of the cubic term, or the one of the replica-symmetry- breaking quartic term, may go through zero. Around such points new spin glass phases are found.
\end{abstract}

75.10 Nr, 75.30 Fv, 75.50 Lk

\section{INTRODUCTION}

Prototypes of systems exhibiting a spin glass phase are the impure metals $\mathrm{Cu}_{1-c} \mathrm{Mn}_{c}$ and $\mathrm{Au}_{1-c} \mathrm{Fe}_{c}$, and the insulator $\mathrm{Eu}_{1-c} \mathrm{Sr}_{c} \mathrm{~S}$. [1] [2] Their common property is frustration of magnetic bonds, which arises from the combination of site disorder and the presence of both ferromagnetic and antiferromagnetic exchange constants. In metals the exchange is the long range, oscillating RKKY interaction; in an insulating SG the interaction is often antiferromagnetic for nearest neighbors and ferromagnetic for next nearest neighbors, while vanishing for more distant pairs.

Edwards and Anderson proposed to describe the spinglass phase by spins with fully random couplings [3]. The mean field limit of this model for Ising spins is the Sherrington-Kirkpatrick (SK) model [4]. The diluted version, where only a fraction of the random bonds is present, is called the Viana-Bray model 5 .

The mean field structure of the SG-phase was derived by Parisi and coworkers. There are infinitely many thermodynamic states, and the order parameter is a function $q(x)$ or $P(q)$, which describes the probabilities of overlaps between pairs of states. [6]

The calculation of corrections to mean field theory has been very laborious. For a recent review, see [7]

The question what remains of mean field theory in low dimensions is still fiercely debated. Nevertheless, the best numerical simulations done so far are well analyzed within a mean field picture [8].

Mean field notions such as branching ratios and ultrametric organization of states have turned out useful in the interpretation of experiments. Indeed, noise experiments on mesoscopic metallic spin glasses have supported the mean field picture [9].

We are thus at the stage where theoretical results are mainly gained from random bond systems, while experiments are mostly performed on random site systems. The understanding of the spin glass phase in random site systems has long been a challenge for the field. Some time ago one of the authors formulated a field theoretic approach for this purpose [10]. In certain limit close analogies were established with known spin glass phases in well known models, such as the Sherrington-Kirkpatrick model, the Hopfield model, and the Viana-Bray model.

The first purpose of the present work is to provide the details of that work. This will be done in section 2 . In section 3 one of the results will be rederived via the Gaussian variational approach. In section 4 we consider a related model with combined bond- and site disorder. In section 5 we extend the approach of section 2 to vector spins. In section 6 we analyze the equations of section 2 for low $T$ and find that replica symmetry must always be broken.

More recently we have considered the Ginzburg-Landau theory of site-disordered spin glasses [11]. It was argued that the prefactor of the cubic or most relevant quartic term may vanish at special points and then change sign. Around such points new types of spin glass phases occur. In section 7 we shall provide the details of that approach. In section 8 we close with a summary. 


\section{THERMODYNAMICS AS A SET OF COUPLED ORDER PARAMETERS}

We discuss a field theory for a random magnet with site-disorder. First we consider a system of Ising spins with Hamiltonian

$$
\mathcal{H}(s)=-\frac{1}{2} \sum_{r r^{\prime}} J\left(r-r^{\prime}\right) s_{r} s_{r^{\prime}} c_{r} c_{r^{\prime}}-H \sum_{r} s_{r} c_{r}
$$

with sums over all $N$ sites of a regular lattice in $d$ dimensions, with periodic boundary conditions. The $c_{r}$ are random occupation numbers; $c_{r}=1$ indicates that at site $r$ a spin is present, whereas $c_{r}=0$ when this site is empty. We shall consider uncorrelated disorder with a fraction $c$ and a total number $c N$ of spins present, with $0<c<1$. $J_{r r^{\prime}}=J\left(r-r^{\prime}\right)$ is a translationally invariant pair coupling, a well known example being the RKKY coupling of metallic spin glasses, $J(r) \sim \cos \left(2 k_{F} r\right) / r^{3}$. However, we can consider more general situations. In a metallic SG there may be additional short-distance (anti-) ferromagnetic clustering [1]. For many insulating spin glasses $J(r)$ takes the value $J_{n n}>0$ for nearest neighbors and $J_{n n n}<0$ for next-nearest neighbors (nnn), while it is effectively zero for more distant pairs.

In the following we shall focus on the situation where for large $c$ ferromagnetic (FM) ordering occurs. For describing two-sublattice antiferromagnetic ordering one first has to make the replacement $s_{r} \rightarrow-s_{r}$ on one of the sublattices. This will redefine the couplings $J$ and introduce the staggered external field $\pm H$. Since our spins are classical, this leads to a problem similar to the ferromagnetic one.

In order to study the thermodynamics of this system at temperature $T \equiv 1 / \beta$ we express the Boltzmann factor as a multiple integral

$$
\exp \{-\beta \mathcal{H}(s)\}=\int D \phi \exp \{-\beta \mathcal{H}(\phi, s)\}
$$

where

$$
D \phi=\operatorname{det}(\beta J / 2 \pi+i 0)^{1 / 2} \prod_{r} \int d \phi_{r}
$$

The integrations run from $-\sqrt{i} \infty$ to $\sqrt{i} \infty$. To assure convergence of the Gaussian integrals, a regulator $i 0$ has been added to $J$; it will from now on not be written explicitely. The partition sum can thus be represented as trace over both discrete $\left(s_{r}\right)$ and continuous $\left(\phi_{r}\right)$ degrees of freedom

$$
Z=\int D \phi \sum_{\{s\}} \exp \{-\beta \mathcal{H}(\phi, s)\}
$$

with Hamiltonian

$$
\beta \mathcal{H}(\phi, s)=\frac{T}{2} \sum_{r r^{\prime}} \phi_{r} J_{r r^{\prime}}^{-1} \phi_{r^{\prime}}-\sum_{r} c_{r}\left\{\phi_{r}+h\right\} s_{r}
$$

where $h=\beta H$.

The quenched averaged free energy follows from $\overline{\log Z}$. The replica method is employed for studying this quantity. One thus calculates the quenched averages $\overline{Z^{n}}$ for $n=1,2,3, \cdots$. The results have to be continued to the limit $n \rightarrow 0$. This procedure introduces replicated field and spin variables $\phi_{r}^{\alpha}, s_{r}^{\alpha}=s_{r}^{\alpha}$, for $1 \leq \alpha \leq n$. The average over the occupation variables $c_{r}$ can now be performed. Since spin sums are decoupled at each site, we may now write $s_{r}^{\alpha}$ as $s_{\alpha}$, which leads to

$$
\overline{Z^{n}}=\int D \phi \exp \left\{-\beta \mathcal{H}_{n}(\phi)\right\}
$$

with replicated Hamiltonian

$$
\beta \mathcal{H}_{n}(\phi)=\frac{T}{2} \sum_{\alpha=1}^{n} \sum_{r r^{\prime}} \phi_{r}^{\alpha} J_{r r^{\prime}}^{-1} \phi_{r^{\prime}}^{\alpha}-c N \Phi_{n}
$$

where 


$$
\Phi_{n}=n \log 2+\frac{1}{c N} \sum_{r} \log \left(1-c+c \operatorname{tr}_{s} \exp \sum_{\alpha=1}^{n}\left(\phi_{r}^{\alpha}+h\right) s_{\alpha}\right)
$$

and where $\operatorname{tr}_{s}$ stands for the normalized sum over $s_{\alpha}= \pm 1,(\alpha=1, \cdots, n)$,

$$
\operatorname{tr}_{s}=\frac{1}{2^{n}} \sum_{\left\{s_{\alpha}= \pm 1\right\}}=\frac{1}{2^{n}} \sum_{s_{1}= \pm 1} \cdots \sum_{s_{n}= \pm 1} .
$$

In order to proceed one has to make an assumption for the order parameter(s). In a mean field approach the magnetization per spin, $M$, will be proportional to the mean field value of $m_{\alpha}=\left[\phi_{\alpha}\right]$, where

$$
[A] \equiv N^{-1} \sum_{r} A_{r}
$$

denotes the spatial average of an observable $A_{r}$. The main step in the present work is to introduce, in the spirit of Edwards-Anderson, also space-independent composite order parameters such as $\left[\phi_{\alpha} \phi_{\beta}\right]_{c}$. This involves the second spatial cumulant

$$
p_{\alpha \beta}=\left[\phi_{\alpha} \phi_{\beta}\right]_{c} \equiv\left[\phi_{\alpha} \phi_{\beta}\right]-\left[\phi_{\alpha}\right]\left[\phi_{\beta}\right]
$$

$m_{\alpha}$ and $p_{\alpha \beta}$ are the first terms in a cumulant expansion in powers of $\phi_{\alpha}$. In Fourier space $m_{\alpha}$ equals $\hat{\phi}_{\alpha}(0)$ and $p_{\alpha \beta}=$ $\sum_{k \neq 0} \hat{\phi}_{\alpha}(k) \hat{\phi}_{\beta}(-k)$. Ferromagnetic ordering implies a macroscopic occupation of the $k=0$ mode, $\hat{\phi}_{\alpha}(0)=\mathcal{O}(1)$, while a spin density wave has a macroscopic occupation of some $k \neq 0$. Spin glass ordering, however, occurs when $p_{\alpha \beta}>0$ for $\alpha \neq \beta$ and is caused by a small occupation of all wavenumbers $k \neq 0$.

In our approach the order parameters explicitly take into account the macroscopic contributions of combinations of Fourier modes. (e.g. $M_{\alpha}=\hat{\phi}_{\alpha}(k=0)$ ). The resulting Fourier sums (e.g. $\sum_{k \neq 0} \hat{\phi}_{\alpha}(k) \hat{\phi}_{\beta}(k)$ ) "cannot bite", as their "teeth" have been removed. Likewise, at third order

$$
\left[\phi_{\alpha} \phi_{\beta} \phi_{\gamma}\right]_{c}=\sum_{k_{1} \neq 0 ; k_{2} \neq 0 ; k_{1} \neq-k_{2}} \hat{\phi}_{\alpha}\left(k_{1}\right) \hat{\phi}_{\beta}\left(k_{2}\right) \hat{\phi}_{\gamma}\left(-k_{1}-k_{2}\right)
$$

only incorporates small contributions from all $k_{1}$ and $k_{1}$, and no macroscopic $\left(M_{\alpha}\right.$ or $\left.q_{\alpha \beta}\right)$ contribution.

For a full description of the problem an infinity of spatial cumulants is needed. We shall, however, first see in how far low order approximations already lead to meaningful results.

\section{Second order cumulant expansion or Gaussian approximation}

As in the derivation of the Fokker-Planck equation, one may hope that the Gaussian approximation already leads to meaningful insights, and does not yet produce unphysical effects such as negative entropies.

After expanding the logarithm of eq. (8) in powers of $c /(1-c)$, we introduce in the $\ell$ 'th term the concentration factor

$$
\gamma_{\ell}=\frac{(-c)^{\ell-1}}{\ell(1-c)^{\ell}} \quad(\ell=1,2,3, \cdots)
$$

and we have to replicate the spins $\ell$ times. This leads to the " $\ell$-spin"

$$
\sigma_{\alpha}=\sum_{j=1}^{\ell} s_{\alpha}^{(j)}
$$

The normalized trace over its values will be denoted by $\operatorname{tr}_{s}^{(\ell)}$. When truncating the cumulant expansion beyond second order, the spatial average leads to

$$
\left[\exp \sum_{\alpha} \phi_{\alpha} \sigma_{\alpha}\right] \equiv \exp \left[\exp \sum_{\alpha} \phi_{\alpha} \sigma_{\alpha}\right]_{c}=\exp \left(\sum_{\alpha} m_{\alpha} \sigma_{\alpha}+\frac{1}{2} \sum_{\alpha \beta} p_{\alpha \beta} \sigma_{\alpha} \sigma_{\beta}\right)
$$


The relation between $p_{\alpha \beta}$ and the $\phi$ 's is imposed by inserting for each set $\alpha \leq \beta$

$$
\begin{aligned}
1 & =\int \mathrm{d} p_{\alpha \beta} \delta\left(p_{\alpha \beta}-\left[\phi_{\alpha} \phi_{\beta}\right]_{c}\right) \\
& =\int_{-\infty}^{\infty} \mathrm{d} p_{\alpha \beta} \int_{-i \infty}^{i \infty} \frac{N c}{4 \pi i} \mathrm{~d} q_{\alpha \beta} \mathrm{e}^{\frac{1}{2} N c q_{\alpha \beta}\left(\left[\phi_{\alpha} \phi_{\beta}\right]_{c}-p_{\alpha \beta}\right)} .
\end{aligned}
$$

A Gaussian form in the $\phi$ 's is then obtained, so they can be integrated out. This yields for the replicated free energy per $\operatorname{spin} \beta F_{n}=(-1 / N c) \ln Z_{n}$ :

$$
\beta F_{n}=\frac{T}{2 c \hat{J}(0)} \sum_{\alpha} m_{\alpha}^{2}+\frac{1}{2 c} \sum_{\alpha} \int \frac{\mathrm{d}^{d} k}{(2 \pi)^{d}}\{\ln (1-c \beta \hat{J}(k) q)\}_{\alpha \alpha}+\frac{1}{2} \sum_{\alpha \beta} q_{\alpha \beta} p_{\alpha \beta}-\Phi_{n},
$$

with

$$
\Phi_{n}=n \log 2+\sum_{\ell=1}^{\infty} \gamma_{\ell}\left(\operatorname{tr}_{s}^{(\ell)} \exp X^{(\ell)}-1\right)
$$

Further,

$$
X^{(\ell)}=\sum_{\alpha}\left(m_{\alpha}+h\right) \sigma_{\alpha}+\frac{1}{2} \sum_{\alpha \beta} p_{\alpha \beta} \sigma_{\alpha} \sigma_{\beta}
$$

The $k$-integral has arisen from $(1 / N) \sum_{k \neq 0}$, where the exclusion of the $k=0$ term is due to the definition of the spatial cumulant. In the thermodynamic limit this exclusion becomes irrelevant. The ensemble averaged free energy per spin now follows as $F=\lim _{n \rightarrow 0} F_{n} / n$, with $F_{n}$ calculated at its saddlepoint. For $c \geq \frac{1}{2}$ the $\ell$-sum in eq. (18) can defined by a Padé resummation.

The magnetization per spin in the replicated system is

$$
\begin{aligned}
M_{\alpha} & \equiv \frac{T}{c \hat{J}(0)} m_{\alpha}=\overline{\left\langle\sigma_{\alpha}\right\rangle} \\
& \equiv \sum_{\ell=1}^{\infty} \gamma_{\ell} \operatorname{tr}_{s}^{(\ell)} \sigma_{\alpha} \exp X^{(\ell)}
\end{aligned}
$$

The physical interpretation of $q$ follows from its mean field equation. This turns out to be very similar to the one introduced by Edwards-Anderson and Sherrington-Kirkpatrick

$$
q_{\alpha \beta}=\overline{\left\langle\sigma_{\alpha} \sigma_{\beta}\right\rangle} \equiv \sum_{\ell=1}^{\infty} \gamma_{\ell} \operatorname{tr}_{s}^{(\ell)} \sigma_{\alpha} \sigma_{\beta} \exp X^{(\ell)}=\sum_{\ell=1}^{\infty} \gamma_{\ell} \frac{\operatorname{tr}_{s}^{(\ell)} \sigma_{\alpha} \sigma_{\beta} \exp X^{(\ell)}}{\operatorname{tr}_{s}^{(\ell)} \exp X^{(\ell)}}
$$

The last equality holds since the denominator equals unity in the replica limit $n \rightarrow 0$. For $c \rightarrow 0$ only the $\ell=1$ term $\overline{\left\langle s_{\alpha} s_{\beta}\right\rangle}$ survives and one exactly recovers the two state overlap introduced by Edwards-Anderson and SherringtonKirkpatrick. Variation with respect to $q_{\alpha \beta}$ yields

$$
p_{\alpha \beta}=\int \frac{\mathrm{d}^{d} k}{(2 \pi)^{d}}\left(\frac{\beta \hat{J}(k)}{1-c \beta \hat{J}(k) q}\right)_{\alpha \beta}
$$

This form exhibits clustering effects. Indeed, decomposing $q_{\alpha \beta}=q_{d} \delta_{\alpha \beta}+\tilde{q}_{\alpha \beta}$ with $q_{d}=q_{\alpha \alpha}$ and $\tilde{q}_{\alpha \alpha}=0$, we may also write for $\alpha \neq \beta$

$$
\begin{aligned}
p_{\alpha \beta} & =\int \frac{\mathrm{d}^{d} k}{(2 \pi)^{d}} \beta \hat{J}_{\mathrm{eff}}(k)\left(\frac{1}{1-c \beta \hat{J}_{\mathrm{eff}}(k) q}\right)_{\alpha \beta} \\
& =\int \frac{\mathrm{d}^{d} k}{(2 \pi)^{d}} c \beta^{2} \hat{J}_{\mathrm{eff}}^{2}(k)\left(\frac{\tilde{q}}{1-c \beta \hat{J}_{\mathrm{eff}}(k) q}\right)_{\alpha \beta} \quad(\alpha \neq \beta),
\end{aligned}
$$


with the effective coupling

$$
\hat{J}_{\mathrm{eff}}(k)=\frac{\hat{J}(k)}{1-c \beta \hat{J}(k) q_{d}} .
$$

Expansion in powers of $c$ indeed shows that it is a multi-spin effect. Maxima of $\hat{J}$ are more pronounced in $\hat{J}_{\text {eff }}$. This describes the formation of finite clusters that are precursors of the would-be spin density wave or (anti-) ferromagnetic phase transition. In metallic spin glasses incomplete spin density waves have indeed been observed by neutron scattering. [12] It is well known that both in metallic and in insulating spin glasses there appear ferromagnetic clusters near the ferromagnetic transition line; these clusters (sometimes called "fat spins") act as a quite rigid effective spins, that may contain up to 2000 magnetic atoms, making quite visible moves. They are responsible for the dynamics in the cluster glass phase [1]. We shall return to this point in Section VII.

(At low temperature this expression for $J_{\text {eff }}$ becomes singular, though eq. (23) is well behaved. It is then more appropriate to interpret $\hat{J} /\left(1-c \beta \hat{J}\left(q_{d}-q_{E A}\right)\right)$ as the effective coupling, where $q_{E A}=\max q_{\alpha \neq \beta}$ is the EdwardsAnderson order parameter).

\section{Replica symmetry}

To obtain an idea of the content of previous expressions, we consider them in the replica-symmetric sector. The free energy then reads

$$
\begin{aligned}
\beta F & =\frac{T}{2 c \hat{J}(0)} m^{2}+\frac{1}{2 c} \int \frac{\mathrm{d}^{d} k}{(2 \pi)^{d}}\left\{\ln \left(1-c \beta \hat{J}(k)\left(q_{d}-q\right)\right)-\frac{c \beta \hat{J}(k)}{1-c \beta \hat{J}(k)\left(q_{d}-q\right)}\right\} \\
& +\frac{1}{2}\left(p_{d} q_{d}-p q\right)-\sum_{\ell} \gamma_{\ell} \int g(x) \mathrm{d} x \ln \int g(y) \mathrm{d} y 2^{\ell} \cosh ^{\ell}\left(h+m+x \sqrt{p}+y \sqrt{p_{d}-p}\right),
\end{aligned}
$$

where

$$
g(x)=\frac{e^{-x^{2} / 2}}{\sqrt{2 \pi}}
$$

is the Gaussian weight. The saddle point equations read

$$
\begin{aligned}
p & =\int \frac{\mathrm{d}^{d} k}{(2 \pi)^{d}} \frac{c \beta^{2} \hat{J}^{2}(k)}{\left(1-c \beta \hat{J}(k)\left(q_{d}-q\right)\right)^{2}}, \\
p_{d} & =p+\int \frac{\mathrm{d}^{d} k}{(2 \pi)^{d}} \frac{\beta \hat{J}(k)}{1-c \beta \hat{J}(k)\left(q_{d}-q\right)} \\
q & =\sum_{\ell} \gamma_{\ell} \int g(x) \mathrm{d} x \ell^{2}\left(\frac{\int g(y) \mathrm{d} y \cosh ^{\ell} \psi \tanh \psi}{\int g(y) \mathrm{d} y \cosh ^{\ell} \psi}\right)^{2}, \\
q_{d} & =\sum_{\ell} \gamma_{\ell} \int g(x) \mathrm{d} x \frac{\int g(y) \mathrm{d} y \cosh ^{\ell} \psi\left(\ell+\ell(\ell-1) \tanh ^{2} \psi\right)}{\int g(y) \mathrm{d} y \cosh ^{\ell} \psi}
\end{aligned}
$$

where

$$
\psi=h+m+x \sqrt{\pi}+y \sqrt{p_{d}-p} .
$$

These expressions will be used later on for small $T$, where the cosh's essentially become exponentials.

\section{Relation to the Hopfield model and the Sherrington-Kirkpatrick model}

Despite of the simplifications made, the above expressions are very rich. In some limits of long range couplings they become exact. 
Consider the situation where

$$
J(k)=J_{0} \quad k_{0}<|k|<k_{1}
$$

and zero outside this shell. This corresponds to a long range spatial coupling

$$
J(r)=J_{0} \int_{k_{0}<k<k_{1}} \frac{\mathrm{d}^{d} k}{(2 \pi)^{d}} \frac{\sin (k r)}{k r} .
$$

We denote the volume in phase space of this shell by $\Delta=\int_{k_{0}}^{k_{1}} \mathrm{~d}^{d} k /(2 \pi)^{d}$. Consider the combined limit $c \rightarrow 0, k_{1} \rightarrow k_{0}$, such that $\alpha=\Delta / c$ remains fixed. Inserting this in eq. 117) we obtain for its spin glass content $(m=0)$

$$
\beta F_{n}=\frac{\alpha}{2} \sum_{\alpha}\left\{\log \left(1-c \beta J_{0} q\right)\right\}_{\alpha \alpha}+\frac{1}{2} \sum_{\alpha \beta} q_{\alpha \beta} p_{\alpha \beta}-\Phi_{n} .
$$

In order to understand its meaning, let us consider the case of replica symmetry. We get in the limit $n \rightarrow 0$

$$
\begin{aligned}
\beta F= & \frac{\alpha}{2}\left\{\log \left(1-c \beta J_{0}(1-q)\right)-\frac{c \beta J_{0} q}{1-c \beta J_{0}(1-q)}\right\}+\frac{1}{2} p(1-q) \\
& -\int \frac{d x}{\sqrt{2 \pi}} e^{-x^{2} / 2} \log 2 \cosh x \sqrt{p}
\end{aligned}
$$

with $p$ and $q$ obeying

$$
\begin{aligned}
p & =\frac{\alpha\left(c \beta J_{0}\right)^{2} q}{\left(1-c \beta J_{0}(1-q)\right)^{2}}, \\
q & =\int_{-\infty}^{\infty} \frac{\mathrm{d} x}{\sqrt{2 \pi}} e^{-x^{2} / 2} \tanh ^{2} x \sqrt{p} .
\end{aligned}
$$

After a rescaling $\left(T \rightarrow c J_{0} \tilde{T}, p \rightarrow \alpha r / \tilde{T}^{2}\right)$ these are exactly the replica-symmetric free energy and its saddle point equations for the spin glass phase in the Hopfield model of a neural network with $\alpha N$ patterns [13]. More generally, eq. (32) is the free energy including replica symmetry breaking. In the limit $\Delta \rightarrow 0, c \rightarrow 0$, with fixed $\Delta / c=\alpha$ one considers long range interactions between widely separated spins, that effectively lead to random interactions. It has been checked that loop-corrections do not contribute in the limit. So (32) is an exact mean field theory in this long range limit. Note that this may occur in any fixed dimension $(d=1,2,3, \cdots)$.

The SK-model arises in the subsequent limit $\alpha \rightarrow \infty$. To show this we must rescale $T \rightarrow \tilde{T} c J_{0} \sqrt{\alpha}$. Then the first relation in eq. (34) becomes linear, and eq. (32) and (33) become linear+quadratic in $q_{\alpha \beta}$, so that $p_{\alpha \beta}$ and $q_{\alpha \beta}$ are linearly related. After elimination the $p$ 's, we recover expressions that in the limit $n \rightarrow 0$ are equivalent to the SK model. Hereto we also use that for small $n$ our expression $-1+\operatorname{tr}_{s} \exp \left(p_{\alpha \beta} s_{\alpha} s_{\beta} / 2\right)$ is proportional to $n$ and to this order equivalent to the SK result $\log \operatorname{tr}_{s} \exp \left(p_{\alpha \beta} s_{\alpha} s_{\beta} / 2\right)$.

The present random site problem was also studied, for the case of Heisenberg spins, by Bray and Moore [14]. These authors do not introduce conjugated spin variables but make a cumulant expansion of the Boltzmann factor up to second order in the occupation factors $c_{i}$. Since the latter are zero or one, there is, however, no reason to stop at second order. Therefore the results differ from ours and are incorrect. For instance, according to ref. 14] the spin glass transition temperature scales as $T_{g} \sim c$ for small $c$ both for short range and RKKY couplings. We find, however, from eq. (34) that $T_{g}=J_{R} \sim \sqrt{c}$. Below we shall derive $T_{g} \sim c$ for RKKY couplings via a more intricate analysis, that takes into account more contributions.

\section{Full cumulant expansion: the direct way}

The above results do not exhibit a percolation transition. Indeed, $T_{s g} \sim \sqrt{c}$ does not vanish identically for small enough concentrations. This is related to the fact that the percolation transition of systems with $z=\infty$ occurs at $c=0$. Also for RKKY couplings the present prediction overestimates $T_{g}$. Here one expects the well established "concentration scaling" $T_{s g} \sim c$. []

We therefore consider the full cumulant expansion of eq. (8). We introduce order parameters

$$
p_{\alpha_{1} \cdots \alpha_{k}}=\left[\phi_{\alpha_{1}} \cdots \phi_{\alpha_{k}}\right]_{c}
$$


as well as conjugated parameters $q_{\alpha_{1} \cdots \alpha_{k}}$. They are symmetric in their replica indices. The replicated free energy may be written as

$$
\beta F_{n}=-\Psi_{n}(q)+p q-\Phi_{n}(p)
$$

where

$$
\Psi_{n}=\frac{1}{c N} \log \int D \phi \exp \left(-\frac{T}{2} \sum_{\alpha r r^{\prime}} \phi_{r}^{\alpha} J_{r r^{\prime}}^{-1} \phi_{r^{\prime}}^{\alpha}+c N \sum_{k=1}^{\infty} \frac{1}{k !} \sum_{\alpha_{1} \cdots \alpha_{k}} q_{\alpha_{1} \cdots \alpha_{k}}\left[\phi_{\alpha_{1}} \cdots \phi_{\alpha_{k}}\right]_{c}\right)
$$

and

$$
p q=\sum_{k=1}^{\infty} \frac{1}{k !} \sum_{\alpha_{1} \cdots \alpha_{k}} p_{\alpha_{1} \cdots \alpha_{k}} q_{\alpha_{1} \cdots \alpha_{k}}
$$

$\Phi_{n}$ is given by eq. (18) with

$$
X^{(\ell)}=\sum_{\alpha}\left(p_{\alpha}+h\right) \sigma_{\alpha}+\frac{1}{2 !} \sum_{\alpha \beta} p_{\alpha \beta} \sigma_{\alpha} \sigma_{\beta}+\frac{1}{3 !} \sum_{\alpha \beta \gamma} p_{\alpha \beta \gamma} \sigma_{\alpha} \sigma_{\beta} \sigma_{\gamma}+\cdots
$$

The free energy is now determined by the infinite set of coupled equations

$$
\begin{aligned}
p_{\alpha_{1} \cdots \alpha_{k}} & =k ! \frac{\partial}{\partial q_{\alpha_{1} \cdots \alpha_{k}}} \Psi_{n}(q) \\
q_{\alpha_{1} \cdots \alpha_{k}} & =k ! \frac{\partial}{\partial p_{\alpha_{1} \cdots \alpha_{k}}} \Phi_{n}(p) .
\end{aligned}
$$

\section{A resummation}

The above result is not very useful at low temperatures, where the $p$ 's become very large. However, it is possible to resum the contributions. This can be performed in the following elegant way. In the $\ell$ 'th term of $\Phi_{n}$ there are spin variables $s_{\alpha}^{(j)},(j=1, \cdots, \ell)$, which we now label as $s_{a}$ with $a=(\alpha, j)$. Though they do not depend on $j$, we also denote $\phi_{\alpha}$ as $\phi_{a}$. We start from the definition

$$
\left[\exp \sum_{a} \phi_{\alpha} s_{a}\right]=\exp \left(\left[\exp \sum_{a} \phi_{\alpha} s_{a}\right]_{c \cdot-1}\right)
$$

The obvious relation

$$
\left[A+A_{a} \phi_{r}^{a}+A_{a b} \phi_{r}^{a} \phi_{r}^{b}+A_{a b c} \phi_{r}^{a} \phi_{r}^{b} \phi_{r}^{c}+\cdots\right]_{c}=A+A_{a}\left[\phi^{a}\right]_{c}+A_{a b}\left[\phi^{a} \phi^{b}\right]_{c}+A_{a b c}\left[\phi^{a} \phi^{b} \phi^{c}\right]_{c}+\cdots
$$

(summation convention employed) defines cumulants of any expandable local function of $\phi_{r}^{a}$. It is a generalization of the definition

$$
\left[\mathrm{e}^{\phi_{a} s_{a}}\right]_{c}=1+s_{a}\left[\phi_{a}\right]_{c}+\frac{1}{2} s_{a} s_{b}\left[\phi_{a} \phi_{b}\right]_{c}+\frac{1}{6} s_{a} s_{b} s_{c}\left[\phi_{a} \phi_{b} \phi_{c}\right]_{c}+\frac{1}{24} s_{a} s_{b} s_{c} s_{d}\left[\phi_{a} \phi_{b} \phi_{c} \phi_{d}\right]_{c}+\cdots
$$

We employ the identity

$$
\exp \left(\sum_{a} \phi_{a} s_{a}\right)=\prod_{a} \cosh \phi_{a}\left(1+s_{a} \tanh \phi_{a}\right) \equiv \prod_{a} c_{a}\left(1+s_{a} t_{a}\right) .
$$

Using the linear property of series of cumulants, eq. (42), this product brings

$$
\begin{aligned}
{\left[\exp \sum_{a} \phi_{a} s_{a}\right]_{c} } & =\left[\prod_{e} c_{e}\right]_{c}+\sum_{a} s_{a}\left[t_{a} \prod_{e} c_{e}\right]_{c}+\frac{1}{2} \sum_{a \neq b} s_{a} s_{b}\left[t_{a} t_{b} \prod_{e} c_{e}\right]_{c} \\
& +\frac{1}{6} \sum_{a \neq b \neq c \neq a} s_{a} s_{b} s_{c}\left[t_{a} t_{b} t_{c} \prod_{e} c_{e}\right]_{c}+\frac{1}{24} \sum_{a, b, c, d ; \text { different }} s_{a} s_{b} s_{c} s_{d}\left[t_{a} t_{b} t_{c} t_{d} \prod_{e} c_{e}\right]_{c}+\cdots
\end{aligned}
$$


That the subtracted terms indeed work out this way can be checked for low orders in the small $\phi_{\alpha}$ expansion. Up to quartic order the right hand side gives

$$
\begin{aligned}
& {\left[1+\frac{1}{2} \phi_{a}^{2}+\frac{1}{8} \phi_{a}^{2} \phi_{b}^{2}-\frac{1}{12} \phi_{a}^{4}\right]_{c}+\left[s_{a} \phi_{a}\left(1-\frac{1}{3} \phi_{a}^{2}+\frac{1}{2} \phi_{b}^{2}\right)\right]_{c}} \\
& +\frac{1}{2}\left[s_{a} s_{b} \phi_{a} \phi_{b}\left(1-\frac{1}{3} \phi_{a}^{2}-\frac{1}{3} \phi_{b}^{2}+\frac{1}{2} \phi_{c}^{2}\right)-\phi_{a}^{2}\left(1-\frac{2}{3} \phi_{a}^{2}+\frac{1}{2} \phi_{c}^{2}\right)\right]_{c} \\
& +\frac{1}{6}\left[s_{a} s_{b} s_{c} \phi_{a} \phi_{b} \phi_{c}-3 s_{a} \phi_{a} \phi_{b}^{2}+2 s_{a} \phi_{a}^{3}\right]_{c} \\
& +\frac{1}{24}\left[s_{a} s_{b} s_{c} s_{d} \phi_{a} \phi_{b} \phi_{c} \phi_{d}-6 s_{a} s_{b} \phi_{a} \phi_{b} \phi_{c}^{2}+3 \phi_{a}^{2} \phi_{b}^{2}+8 s_{a} s_{b} \phi_{a} \phi_{b}^{3}-6 \phi_{a}^{4}\right]_{c}
\end{aligned}
$$

where now the sums over repeated indices are unrestricted. This expression indeed coincides with eq. (43).

For every integer $1 \leq k \leq n$ and $\ell \geq 1$ we introduce symmetric order parameters

$$
p_{\alpha_{1} \cdots \alpha_{k}}^{(\ell)}=\left[c_{1}^{\ell} \cdots c_{n}^{\ell} t_{\alpha_{1}} \cdots t_{\alpha_{k}}\right]_{c}
$$

with the restriction that the total number of replica indices that occur more than once, does not exceed $\ell$. (For example, $p_{\alpha \alpha \beta \gamma}^{(2)}$ is needed, but $p_{\alpha \alpha \alpha \beta \beta \gamma}^{(4)}$ is not). Further

$$
p^{(\ell)}=\left[c_{1}^{\ell} \cdots c_{n}^{\ell}\right]_{c}-1
$$

Introducing the spin variable

$$
X^{(\ell)}=\sum_{a}\left(p_{\alpha}^{(\ell)}+h\right) s_{a}+\frac{1}{2 !} \sum_{a \neq b} p_{\alpha \beta}^{(\ell)} s_{a} s_{b}+\frac{1}{3 !} \sum_{a \neq b \neq c \neq a} p_{\alpha \beta \gamma}^{(\ell)} s_{a} s_{b} s_{c}+\cdots
$$

we find that the $\ell^{\prime}$ th term of $\Phi_{n}$ involves an expression $\operatorname{tr}_{s}^{(\ell)} \exp \left\{p^{(\ell)}+X^{(\ell)}\right\}$. Next we introduce the conjugate order parameters $q^{(\ell)}, q_{\alpha}^{(\ell)}, q_{\alpha \beta}^{(\ell)}, \cdots$, through relations of the type (16),

$$
1=\int_{-\infty}^{\infty} \mathrm{d} p_{\alpha_{1} \cdots \alpha_{k}}^{(\ell)} \int_{-i \infty}^{i \infty} \frac{N c}{k ! 2 \pi i} \mathrm{~d} q_{\alpha_{1} \cdots \alpha_{k}}^{(\ell)} \exp \frac{1}{k !} N c q_{\alpha_{1} \cdots \alpha_{k}}^{(\ell)}\left(\left[t_{\alpha_{1}} \cdots t_{\alpha_{k}} \prod_{e} c_{e}\right]_{c}-p_{\alpha_{1} \cdots \alpha_{k}}^{(\ell)}\right)
$$

Let us first consider the saddle point equations for $p^{(\ell)}$ and $q^{(\ell)}$. It is readily seen that $p^{(\ell)}=\mathcal{O}(n)$ and that $q^{(\ell)}=1+\mathcal{O}(n)$. This implies that $p^{(\ell)}$ can be omitted and that the $q^{(\ell)}$ terms can be summed. These manipulations lead us to a replicated free energy of the form (36),

$$
\beta F_{n}=-\Psi_{n}(q)+(p, q)-\Phi_{n}(p),
$$

with $\Phi_{n}$ given by eqs. (18) and (49). Further,

$$
(p, q) \equiv \sum_{\ell=1}^{\infty} \gamma_{\ell} \sum_{k=1}^{\infty} \frac{1}{k !} \sum_{\alpha_{1} \cdots \alpha_{k}} p_{\alpha_{1} \cdots \alpha_{k}}^{(\ell)} q_{\alpha_{1} \cdots \alpha_{k}}^{(\ell)}
$$

and

$$
\begin{gathered}
\Psi_{n}=\frac{1}{c N} \log \int D \phi \exp \left(-\frac{T}{2} \sum_{\alpha r r^{\prime}} \phi_{r}^{\alpha} J_{r r^{\prime}}^{-1} \phi_{r^{\prime}}^{\alpha}+N\left[\log \left(1-c+c \operatorname{tr}_{s} \exp \sum_{\alpha} \phi_{\alpha} s_{\alpha}\right)\right]_{c}\right) \\
* \exp \left(c N \sum_{\ell=1}^{\infty} \gamma_{\ell} \sum_{k=1}^{\infty} \frac{1}{k !} \sum_{\alpha_{1} \cdots \alpha_{k}} q_{\alpha_{1} \cdots \alpha_{k}}^{(\ell)}\left[c_{1}^{\ell} \cdots c_{n}^{\ell} t_{\alpha_{1}} \cdots t_{\alpha_{k}}\right]_{c}\right) .
\end{gathered}
$$

In this expression the logarithm sums the $q^{(\ell)} \rightarrow 1$ contributions. The difficult part of the problem is still the integration over the $\phi$ fields. One might even think that nothing has been gained in the present representation. However, the dangerous order parameters have been subtracted explicitely. In the remainder of the paper we shall mainly consider situations where $\Psi_{n}$ has a relatively simple form. Nevertheless, we shall be able to draw interesting conclusions.

In general, the $\phi$ integrals cannot be performed exactly. If the exponent is expanded up to second order in $\phi$, one recovers eq. (17). 


\section{Small concentrations and the relation with the Viana-Bray model}

We now consider the limit of small $c$ at fixed $T$. Here only $\ell=1$ contributes, so that all $q$ 's and $p$ 's with $\ell>1$ can be omitted. The remaining ones have all replica indices different from each other. $\Psi_{n}$ can be approximated to order $q^{2}$. The integrals can be performed. To see how this goes, consider eq. (53). First, since $c$ is small, we may omit the term $N[\log \cdots]_{c}$ in the exponent. When expanding in powers of $q$ we have bilinear terms of the type

$$
c^{2} q_{\alpha_{1} \cdots \alpha_{k}} q_{\alpha_{1}^{\prime} \cdots \alpha_{k^{\prime}}^{\prime}} \int D \phi \exp \left(-\frac{T}{2} \sum_{\alpha r r^{\prime}} \phi_{r}^{\alpha} J_{r r^{\prime}}^{-1} \phi_{r^{\prime}}^{\alpha}\right) N\left[c_{1} \cdots c_{n} t_{\alpha_{1}} \cdots t_{\alpha_{k}}\right]_{c} N\left[c_{1} \cdots c_{n} t_{\alpha_{1}^{\prime}} \cdots t_{\alpha_{k^{\prime}}^{\prime}}\right]_{c} .
$$

Neglecting, for the moment, the difference between cumulants and ordinary moments, the integral can be written as

$$
\begin{aligned}
& \sum_{r_{1}, r_{2}} \sum_{s, \tilde{s}} s_{\alpha_{1}} \cdots s_{\alpha_{k}} \tilde{s}_{\alpha_{1}^{\prime}} \cdots \tilde{s}_{\alpha_{k^{\prime}}^{\prime}} \int D \phi \exp \left(-\frac{T}{2} \sum_{\alpha r r^{\prime}} \phi_{r}^{\alpha} J_{r r^{\prime}}^{-1} \phi_{r^{\prime}}^{\alpha}\right) \exp \sum_{\gamma}\left(\phi_{r_{1}}^{\gamma} s_{\gamma}+\phi_{r_{2}}^{\gamma} \tilde{s}_{\gamma}\right) \\
= & \sum_{r_{1}, r_{2}} \sum_{s, \tilde{s}} s_{\alpha_{1}} \cdots s_{\alpha_{k}} \tilde{s}_{\alpha_{1}^{\prime}} \cdots \tilde{s}_{\alpha_{k^{\prime}}^{\prime}} \exp \left(\beta J\left(r_{1}-r_{2}\right) \sum_{\gamma} s_{\gamma} \tilde{s}_{\gamma}\right) .
\end{aligned}
$$

For each fixed $\gamma$, the sum over $s_{\gamma}$ and $\tilde{s}_{\gamma}$ can have preexponential factor $1, s_{\gamma}, \tilde{s}_{\gamma}$, and $s_{\gamma} \tilde{s}_{\gamma}$. When performing the sums, it is readily see that only the first or the last type of terms survive; this explains also why terms linear in $q$ vanish identically. This implies that only diagonal terms in the $q_{\alpha_{1} \cdots \alpha_{k}}$ remain, with prefactors that are spatial sums of $\cosh ^{n} \beta J\left(r_{1}-r_{2}\right) \tanh ^{k} \beta J\left(r_{1}-r_{2}\right)$. Investigating the subtraction terms that occur in the spatial cumulants we observe that replacing the cumulants by ordinary moments leads at this point only to corrections of order $1 / N$, that are negligible in the thermodynamic limit. The same effect led to the exclusion of the $k=0$ term in (17). This supports previous finding that the role of the order parameters is only to eliminate the macroscopic parts of spatial averages; the remainder being the well-behaved cumulants. For small $n$ we find for the replicated free energy

$$
\beta F_{n}=\frac{c}{2}\left\{-n \tau_{0}+\tau_{1} \sum_{\alpha} M_{\alpha}^{2}+\tau_{2} \sum_{\alpha<\beta} q_{\alpha \beta}^{2}+\tau_{3} \sum_{\alpha<\beta<\gamma} q_{\alpha \beta \gamma}^{2}+\cdots\right\}-n \log 2+1-\operatorname{tr}_{s} \exp X
$$

with

$$
\tau_{0}=\sum_{r} \log \cosh \beta J(r) \quad \tau_{k}=\sum_{r} \tanh ^{k} \beta J(r) \quad(k \geq 1)
$$

and

$$
X=\sum_{\alpha}\left(c \tau_{1} M_{\alpha}+h\right) s_{\alpha}+c \tau_{2} \sum_{\alpha<\beta} q_{\alpha \beta} s_{\alpha} s_{\beta}+c \tau_{3} \sum_{\alpha<\beta<\gamma} q_{\alpha \beta \gamma} s_{\alpha} s_{\beta} s_{\gamma}+\cdots
$$

When couplings are (mainly) ferromagnetic, a ferromagnetic transition sets in at temperature such that $c \tau_{1}=1$. If the $J(r)$ are partly positive and negative, there are cancellations in this sum. Then a spinglass phase may occur at a higher temperature. The spinglass phase $\left(q_{\alpha \beta}>0\right)$ sets in at a temperature $T_{g}$ where $c \tau_{2}=1$. If only couplings to $z$ neighbors are different from zero, a SG phase can only occur beyond the percolation threshold $c_{p}=1 / z$. $c / c_{p}>1$ expresses that, on the average, each spin should interact with more than one other spin. For a fcc lattice in $d=3$ this yields $c_{p}=1 / 12 \approx 8.3 \%$, to be compared with the simulated value $c_{p} \approx 13.6 \%$ [15. For the RKKY-coupling in three dimensions, the condition $c \tau_{2}=1$ indeed yields the "concentration scaling" $T_{G} \sim c$ for small $c$.

Some years ago the SK model with diluted random bonds was introduced by Viana and Bray [5]; it is commonly called the Viana-Bray model. These authors also analyzed various aspects of the phase diagram. In an unpublished work one of the present authors [16] derived the same model, without making any analysis of the phase diagram. In the diluted model one assumes that the couplings $J_{r r^{\prime}}$ in eq. (11) vanish with probability $1-p / N$, and are drawn independently from a normalized distribution $r(J)$ with probability $p / N$. In the thermodynamic limit $N \rightarrow \infty$, the parameter $p$ is kept fixed. There is a close analogy with the Flory model of coagulation [17] [18]. The parameter $p$ can be interpreted as a dimensionless time variable, at which the coagulation has been interrupted. For $p<1$ only finite clusters exist, while for $p>1$ an infinite cluster has appeared. On this infinite cluster a spontaneous phase transition can occur, to a (anti-) ferromagnetic or spinglass phase.

It would be interesting to consider $p$ really as a slow time variable, and to consider the combination of very slow cluster growth and spin glass dynamics. In this dynamical model the random couplings would be fixed, but as time 
progresses more and more of them would become active. This idea is inspired on a model, introduced by Coolen, Penney, and Sherrington, where all couplings are active, but their strength changes slowly in time [19].

The quenched free energy of the Viana-Bray model is derived along the following lines. The averaged replicated partition sum equals

$$
\begin{aligned}
\overline{Z^{n}} & =\sum_{\left\{s_{r}^{\alpha}\right\}} \prod_{<r r^{\prime}>}\left\{1-\frac{p}{N}+\frac{p}{N} \int r(J) d J \exp \left(\beta J \sum_{\alpha} s_{r}^{\alpha} s_{r^{\prime}}^{\alpha}\right)\right\} \\
& \approx \sum_{\left\{s_{r}^{\alpha}\right\}} \exp \left\{\frac{p}{2 N} \sum_{r r^{\prime}}\left(-1+\int r(J) d J \cosh ^{n}(\beta J) \prod_{\alpha}\left(1+s_{r}^{\alpha} s_{r^{\prime}}^{\alpha} \tanh \beta J\right)\right)\right\} .
\end{aligned}
$$

Writing out the product over $\alpha$ and decoupling the terms quadratic in products of spins at the same site, one defines

$$
t_{0}=\int r(J) d J \log \cosh \beta J ; \quad t_{k}=\int r(J) d J \tanh ^{k} \beta J \quad(k=1,2,3, \cdots) .
$$

In the limit $n \rightarrow 0$ the replicated free energy takes the form

$$
\beta F_{n}=\frac{p}{2}\left\{-n t_{0}+t_{1} \sum_{\alpha} M_{\alpha}^{2}+t_{2} \sum_{\alpha<\beta} q_{\alpha \beta}^{2}+t_{3} \sum_{\alpha<\beta<\gamma} q_{\alpha \beta \gamma}^{2}+\cdots\right\}-n \log 2-\log \operatorname{tr}_{s} \exp X
$$

with $X$ given by eq. (58) after the replacement $c \tau_{k} \rightarrow p t_{k}$. For a discussion of the phase diagram of this model, see e.g. refs. [5], 20], [21], 22].

For short range couplings in the random site problem the two results are thus closely related. Indeed, since again $-1+\operatorname{tr}_{s} \exp X$ is equivalent to $\log \operatorname{tr}_{s} \exp X$, the only real difference lies in the meaning of the parameters $c \tau_{k}=\left(c / c_{p}\right)\left(\tau_{k} / z\right)=p t_{k}$. In the diluted random bond model $\tau_{k} / z=t_{k}$ is defined as the average of tanh ${ }^{k} \beta J$ over the $^{2}$ distribution of the independent couplings $J$. In the random site model with sure couplings, this average is taken over space.

\section{GAUSSIAN VARIATIONAL APPROACH}

Since disordered systems are so hard to solve, it is becoming more and more popular to investigate variational approaches [23], 24], 25], 26]. In the present problem one starts from eqs. (7) and (8), and chooses the variational Hamiltonian

$$
\mathcal{H}_{\mathrm{var}}=\frac{1}{2} \sum_{i j \alpha \beta}\left(\phi_{i}^{\alpha}-m_{\alpha}\right)\left\{G^{-1}\right\}_{i j}^{\alpha \beta}\left(\phi_{j}^{\beta}-m_{\beta}\right)
$$

The variational propagator $\left\langle\phi_{i}^{\alpha} \phi_{j}^{\beta}\right\rangle_{\mathrm{var}}=G_{i j}^{\alpha \beta}=G_{\alpha \beta}\left(r_{i}-r_{j}\right)$ is translationally invariant and has Fourier transform $\hat{G}_{\alpha \beta}(k)$. The variational free energy per spin becomes

$$
\begin{aligned}
\beta F & =\frac{1}{N c}\left(-\ln Z_{\mathrm{var}}+\left\langle\mathcal{H}_{n}-\mathcal{H}_{\mathrm{var}}\right\rangle_{\mathrm{var}}\right) \\
& =\sum_{\alpha} \frac{m_{\alpha}^{2}}{2 \beta \hat{J}(k=0)}+\frac{1}{2 c} \sum_{\alpha} \int \frac{\mathrm{d}^{d} k}{(2 \pi)^{d}}\left\{-\ln \hat{G}(k)+\frac{\hat{G}(k)}{\beta \hat{J}(k)}\right\}_{\alpha \alpha} \\
& +\sum_{\ell=1}^{\infty} \gamma_{\ell}\left(1-\operatorname{tr}_{\sigma}^{(\ell)} e^{\sum_{\alpha}\left(h+m_{\alpha}\right) \sigma_{\alpha}+\frac{1}{2} \sum_{\alpha \beta} \sigma_{\alpha} G_{\alpha \beta}(r=0) \sigma_{\beta}}\right)
\end{aligned}
$$

The variational equation $\delta F / \delta G_{\alpha \beta}(k)=0$ can be expressed in terms of $q_{\alpha \beta}$ using (22). This yields

$$
G_{\alpha \beta}(k)=\left(\frac{\beta \hat{J}(k)}{1-c \beta \hat{J}(k) q}\right)_{\alpha \beta}
$$

Its $k$-integral can be identified with eq. (23): 


$$
p_{\alpha \beta}=G_{\alpha \beta}(r=0)=\int \frac{\mathrm{d}^{d} k}{(2 \pi)^{d}}\left(\frac{\beta \hat{J}(k)}{1-c \beta \hat{J}(k) q}\right)_{\alpha \beta} .
$$

Due to this, the $\ell$-sum in eq. (63) can be identified with the expressions (18), (19). Therefore the variational equations of the second order cumulant expansion [10] and the Gaussian variational approximation coincide. It is then a small exercise to check that the saddle point value of the free energy also coincides. This does not mean that both approaches are the same. The Gaussian variational Ansatz has looked in a larger space, with variational parameters $G_{\alpha \beta}(r)$, but found the same physics as the second order cumulant expansion with its space-independent parameters $p_{\alpha \beta}$ and $q_{\alpha \beta}$. The reason hereto is simply that both approximations are Gaussian. The equivalence of the two approaches was realized by one of us in fall 1995.

One can go to the continuum limit. Hereto one reinserts the lattice constant $a$, and takes the combined limit $c \rightarrow 0$, $a \rightarrow 0$, such that $\rho=c / a^{d}$ is fixed. The main effect is an overall factor $a^{d}$ (since in the limit $F / a^{d}$ becomes the finite free energy per unit volume) and that only the $\ell=1$ term survives in the sum (because $c \rightarrow 0$ ). This continuum result was discovered independently by Dean and Lancaster [27], who started in the continuum immediately. (We should point out that a proper definition of their path integral would reintroduce a lattice.) They introduce a grand-canonical description of disorder, which is physically the same as the ordinary disorder average, but for their purpose a bit more convenient.

We have criticism to this work. The claim of 27 to present a new field theoretic approach is unjust. Their field theory in replica space (paper I, eq. (4), paper II eq. (2.5)) is just the continuum limit of the above eqs. (6), (7), (8), represented long ago as eqs. (2) and (3) of ref. [10]. The authors of [27] fully refrained from explaining that, despite the different approach, the resulting saddle point equations and saddle point free energy are not new either [28], but coincide with the ones of the second order cumulant expansion of [10], and also discussed above.

\section{A SITE-DISORDERED SPIN GLASS MODEL WITH PAIR OVERLAPS ONLY}

In realistic spin glasses the signs of the interactions oscillate due to the strong distance-dependence for pairs of spins present. We have seen that this leads to global multi-spin order parameters, a somewhat unfamiliar theoretical framework. On the other hand, it is custommary to consider models where the signs are uncorrelated random variables. We shall extend that approach to the site-diluted case, and find that only local pair overlaps occur.

Consider the couplings

$$
J_{r r^{\prime}}=J\left(r-r^{\prime}\right) \xi_{r} \xi_{r^{\prime}}
$$

with independent quenched random numbers $\xi_{r}$. Numerically the simplest choice is $\xi_{r}= \pm 1$ with equal probabilities. We shall take them, however, Gaussian with zero average and unit variance. The function $J(r)$ can now be taken nonnegative. For $J(r) \sim 1 / r^{3}$ this form replaces the deterministic signs of the RKKY interaction by random signs. We proceed along the lines of section 2, and introduce fields $\phi_{r}^{\alpha}$. The disorder average over the random spin configurations and the random $\xi$ 's will now yield

$$
\overline{\exp \left(\sum_{\alpha} s_{\alpha} c_{r}\left(\phi_{r}^{\alpha} \xi_{r}+h\right)\right)}=1-c+c \exp \left(\frac{1}{2} \sum_{\alpha \beta} \phi_{r}^{\alpha} \phi_{r}^{\beta} s_{\alpha} s_{\beta}+h \sum_{\alpha} s_{\alpha}\right)
$$

We can introduce the composite local field $p_{\alpha \beta}(r)=\phi_{r}^{\alpha} \phi_{r}^{\beta}$ as new variable by inserting $\delta$ functions at each lattice site. In their plane wave representations there appear only Gaussian $\phi$ integrals. Integrating them out one is left with a field theory for the space-dependent fields $p_{\alpha \beta}(r), q_{\alpha \beta}(r)$. Its Hamiltonian reads

$$
\beta \mathcal{H}_{n}=\sum_{r} \beta \mathcal{H}(m(r), p(r), q(r))
$$

with

$$
\begin{aligned}
\beta \mathcal{H}(m, p, q) & =\frac{T}{2 c \hat{J}(0)} \sum_{\alpha} m_{\alpha}^{2}+\frac{1}{2 c} \sum_{\alpha}\{\log (1-c \beta \hat{J}(k) q)\}_{r \alpha ; r \alpha}+\frac{1}{2} c \sum_{\alpha \beta} q_{\alpha \beta} p_{\alpha \beta} \\
& -\ln \left(1-c+c \operatorname{tr}_{s} \exp \left(\frac{1}{2} \sum_{\alpha \beta} p_{\alpha \beta} s_{\alpha} s_{\beta}+h \sum_{\alpha} s_{\alpha}\right)\right)
\end{aligned}
$$


Note that the log-term is non-local. In the mean field approximation, the functions $p$ and $q$ are space- independent. The resulting expression is then very close to the one in the second order cumulant expansion of section 2 . Now the spin sums are simpler, since the logarithm needs not be expanded. Consequently, the sign changes in the coefficients $w$ and $y_{1}, y_{2}, y_{3}$, to be discussed in section VII, do not occur in the present model.

As in section 2, eq. (25), the effective coupling is

$$
\hat{J}_{\text {eff }}(k)=\frac{\hat{J}(k)}{1-c \beta \hat{J}(k)\left(1-q_{E A}\right)},
$$

which again exhibits clustering effects.

\section{VECTOR SPINS}

Experimentally, spinglasses usually consist of Heisenberg spins with weak or strong anisotropy. It is therefore useful to extend our formalism to $m$-component spins. We consider the Hamiltonian

$$
\mathcal{H}(s)=-\frac{1}{2} \sum_{\mu \nu r r^{\prime}} J_{r r^{\prime}}^{\mu \nu} S_{r}^{\mu} S_{r^{\prime}}^{\nu} c_{r} c_{r^{\prime}}-\sum_{\mu r} H_{r}^{\mu} S_{r}^{\mu} c_{r}
$$

where $|\mathbf{S}|=1$. The translationally invariant coupling $J$ may contain effects of anisotropy, such as dipolar anisotropy.

\section{Second order cumulant expansion}

Proceeding as above we can carry out the calculations in the second order cumulant expansion. We find

$$
\beta F_{n}=\frac{\beta}{2 c} \sum_{\alpha \mu \nu} \hat{J}^{\mu \nu}(0) M_{\alpha}^{\mu} M_{\beta}^{\nu}+\frac{1}{2 c} \int_{k} \sum_{\alpha \mu}\{\log (1-c \beta \hat{J}(k) q)\}_{\alpha \mu ; \alpha \mu}+\frac{1}{2} \sum_{\alpha \beta \mu \nu} p_{\alpha \beta}^{\mu \nu} q_{\alpha \beta}^{\mu \nu}-\Phi_{n} .
$$

The expression for $\Phi_{n}$ takes the form

$$
\Phi_{n}=n \log \Omega_{m}+\sum_{\ell=1}^{\infty} \gamma_{\ell}\left\{-1+\operatorname{tr}_{S}^{(\ell)} \exp X^{(\ell)}\right\} .
$$

Here $\Omega_{m}$ is the area of a unit sphere in $m$ dimensions $\left(\Omega_{1}=2, \Omega_{2}=2 \pi ; \Omega_{3}=4 \pi\right) . D \mathbf{S}=\Omega_{m}^{-n} d \mathbf{S}_{1} \cdots d \mathbf{S}_{n}$, is the angular integration measure for one replicated spin and $\operatorname{tr}_{S}^{(\ell)}=\prod_{j=1}^{\ell} \int D \mathbf{S}^{(j)}$ is the integration over all replicated spins. Finally,

$$
X^{(\ell)}=\sum_{\alpha \mu}\left(m_{\alpha}^{\mu}+h^{\mu}\right) \sum_{j=1}^{\ell} S_{\alpha}^{(j) \mu}+\frac{1}{2} \sum_{\alpha \beta \mu \nu} p_{\alpha \beta}^{\mu \nu} \sum_{j j^{\prime}=1}^{\ell} S_{\alpha}^{(j) \mu} S_{\beta}^{\left(j^{\prime}\right) \nu} .
$$

In the SK-type limit discussed above, one again obtains and expression quadratic in the $q$ 's:

$$
\beta F_{n}=\frac{\beta}{2 c} \sum_{\alpha \mu \nu r} J_{r 0}^{\mu \nu} M_{\alpha}^{\mu} M_{\alpha}^{\nu}+\frac{\beta^{2}}{4 c} \sum_{r} \sum_{\alpha \beta} \sum_{\mu \nu \rho \sigma} J_{0 r}^{\sigma \mu} J_{r 0}^{\nu \rho} q_{\alpha \beta}^{\mu \nu} q_{\beta \alpha}^{\rho \sigma}+\frac{1}{2} \sum_{\alpha \beta \mu \nu} p_{\alpha \beta}^{\mu \nu} q_{\alpha \beta}^{\mu \nu}-\Phi_{n} .
$$

For isotropic couplings in the presence of an external field, Gabay and Toulouse [29] already showed the occurrence of an irreversibility line where transverse components of the spins freeze.

\section{The resummed cumulant expansion}

Here our aim is to derive the equivalent for vector spins of the resummed cumulant expansion of section II 5 . This will yield, in particular, a prediction for the transition temperature at low concentrations.

The first step in the derivation is the vector analog of the cumulant generating function eq. (41): 


$$
\left[\exp \sum_{a \mu} \phi_{\alpha}^{\mu} S_{a}^{\mu}\right]=\exp \left(\left[\exp \sum_{a \mu} \phi_{\alpha}^{\mu} S_{a}^{\mu}\right]_{c}-1\right)
$$

where $\mu=1,2, \cdots, m$ and where $a=(\alpha, j)$ with $\alpha=1,2, \cdots, n$ and $j=1,2, \cdots, \ell$. The natural generalization of eq. (44) is to introduce

$$
\exp \sum_{\mu} \phi_{\alpha}^{\mu} S_{a}^{\mu}=C_{a}+C_{a} T_{\alpha}\left(\mathbf{S}_{a}\right)=P_{a} \exp \sum_{\mu} \phi_{\alpha}^{\mu} S_{a}^{\mu}+Q_{a} \exp \sum_{\mu} \phi_{\alpha}^{\mu} S_{a}^{\mu},
$$

where

$$
C_{a}=\frac{1}{\Omega_{m}} \int d \mathbf{S}_{a} \exp \sum_{\mu} \phi_{\alpha}^{\mu} S_{a}^{\mu} \equiv P_{a} \exp \sum_{\mu} \phi_{\alpha}^{\mu} S_{a}^{\mu}
$$

with $P_{a}=\Omega_{m}^{-1} \int d \mathbf{S}_{a}$ being a projector, $Q_{a}=1-P_{a}$ being its complement, and

$$
T_{\alpha}\left(\mathbf{S}_{a}\right)=\frac{\exp \sum_{\mu} \phi_{\alpha}^{\mu} S_{a}^{\mu}}{C_{\alpha}}-1
$$

In analogy with section II 5 we define order parameter functions

$$
p_{\alpha_{1} \cdots \alpha_{k}}^{(\ell)}\left(\mathbf{S}_{a_{1}}, \cdots, \mathbf{S}_{a_{k}}\right)=\left[C_{1}^{\ell} \cdots C_{n}^{\ell} T_{\alpha_{1}}\left(\mathbf{S}_{a_{1}}\right) \cdots T_{\alpha_{k}}\left(\mathbf{S}_{a_{k}}\right)\right],
$$

where the total number of repeated replica indices should not exceed $\ell$. Note that this quantity depends only on $k$ spin variables, generalizing a similar property of the combination $p_{\alpha_{1} \cdots \alpha_{k}} s_{a_{1}} \cdots s_{a_{k}}$ of section II 5. The expression for $\Phi_{n}$ takes the form (73) with

$$
X^{(\ell)}=\sum_{a}\left\{p_{\alpha}^{(\ell)}\left(\mathbf{S}_{a}\right)+\mathbf{h} \cdot \mathbf{S}_{a}\right\}+\frac{1}{2 !} \sum_{a \neq b} p_{\alpha \beta}^{(\ell)}\left(\mathbf{S}_{a}, \mathbf{S}_{b}\right)+\frac{1}{3 !} \sum_{a \neq b \neq c \neq a} p_{\alpha \beta \gamma}^{(\ell)}\left(\mathbf{S}_{a}, \mathbf{S}_{b}, \mathbf{S}_{c}\right)+\cdots
$$

Going through similar steps as before we derive the replicated free energy

$$
\beta F_{n}=-\Psi_{n}(q)+p q-\Phi_{n}(p),
$$

where

$$
\begin{aligned}
\Psi_{n}= & \frac{1}{c N} \log \int D \phi \exp \left(-\frac{T}{2} \sum_{\alpha \mu r} \phi_{\alpha}^{\mu}(r)\left\{J^{-1} \phi_{\alpha}\right\}^{\mu}(r)+N\left[\log \left(1-c+c \int D \mathbf{S} \exp \sum_{\alpha} \phi_{\alpha}^{\mu} S_{\alpha}^{\mu}\right)\right]_{c}\right) \\
& * \exp \left(c N \sum_{\ell=1}^{\infty} \gamma_{\ell} \sum_{k=1}^{\infty} \frac{1}{k !} \sum_{\alpha_{1} \cdots \alpha_{k}} P_{1} \cdots P_{k} q_{\alpha_{1} \cdots \alpha_{k}}^{(\ell)}\left(\mathbf{S}_{1}, \cdots, \mathbf{S}_{k}\right)\left[C_{1}^{\ell} \cdots C_{n}^{\ell} T_{\alpha_{1}}\left(\mathbf{S}_{1}\right) \cdots T_{\alpha_{k}}\left(\mathbf{S}_{k}\right)\right]_{c}\right) .
\end{aligned}
$$

and where

$$
p q=\sum_{\ell=1}^{\infty} \gamma_{\ell} \sum_{k=1}^{\infty} \frac{1}{k !} \sum_{\alpha_{1} \cdots \alpha_{k}} P_{1} \cdots P_{k} p_{\alpha_{1} \cdots \alpha_{k}}^{(\ell)}\left(\mathbf{S}_{1}, \cdots, \mathbf{S}_{k}\right) q_{\alpha_{1} \cdots \alpha_{k}}^{(\ell)}\left(\mathbf{S}_{1}, \cdots, \mathbf{S}_{k}\right) .
$$

In the limit of strong anisotropy, where the vector spins are Ising-like and point along the $\pm z$ axis, these equations reduce to the results of section [I 5. Indeed, it can be seen that $q_{\alpha_{1} \cdots \alpha_{k}}^{(\ell)}\left(\mathbf{S}_{1}, \cdots, \mathbf{S}_{k}\right) \rightarrow\left\{q_{\alpha_{1} \cdots \alpha_{k}}^{(\ell)}\right\}_{\text {Ising }} S_{1}^{z} \cdots S_{k}^{z}$ in this limit.

\section{The spin glass transition at low concentrations}

The spin glass transition occurs for small $p_{\alpha \beta}^{(\ell)}$ and $q_{\alpha \beta}^{(\ell)}$. We now restrict ourselves to the case of small $c$, where only the $\ell=1$ terms contribute. The transition temperature is found by considering a replica symmetric solution

$q_{\alpha \beta}\left(\mathbf{S}_{1}, \mathbf{S}_{2}\right)=q_{S G}\left(\mathbf{S}_{1}, \mathbf{S}_{2}\right)$. By expanding the mean field equations of $p_{\alpha \beta}$ and $q_{\alpha \beta}$ it is seen that the condition for the critical point has the form of an integral equation 


$$
q_{S G}\left(\mathbf{S}_{1}, \mathbf{S}_{2}\right)=c \sum_{r} \frac{P_{3} P_{4} q_{S G}\left(\mathbf{S}_{3}, \mathbf{S}_{4}\right) Q_{1} Q_{2} Q_{3} Q_{4} \exp \left(\sum_{\mu \nu}\left\{S_{1}^{\mu} \beta \tilde{J}_{0 r}^{\mu \nu} S_{3}^{\nu}+S_{2}^{\mu} \beta \tilde{J}_{0 r}^{\mu \nu} S_{4}^{\nu}\right\}\right)}{\left\{P_{5} P_{6} \exp \left(\sum_{\mu \nu} S_{5}^{\mu} \beta \tilde{J}_{0 r}^{\mu \nu} S_{6}^{\nu}\right)\right\}^{2}},
$$

where $\tilde{J}_{0 r}^{\mu \nu}=\frac{1}{2}\left(J_{0 r}^{\mu \nu}+J_{r 0}^{\nu \mu}\right)$. Note that this equation has the form eigenvalue equation, Kernel $* q_{S G}=\frac{1}{c} q_{S G}$. This prediction is useful for long range spin glasses with a small concentration of spins, as typically occurs in experiments on metallic spinglasses and also in the Monte Carlo experiment of ref. 30].

The fact that an integral equation occurs is not unexpected, since this is already known to happen for a linear chain. Because of this reason, the analysis of the SG phase for very diluted vector spins is more cumbersome than for Ising spins.

For isotropic couplings, $J^{\mu \nu}(r)=\delta_{\mu \nu} J(r)$ the relevant solution of eq. (85) has the form $q_{S G}\left(\mathbf{S}_{1}, \mathbf{S}_{2}\right)=\mathbf{S}_{1} \cdot \mathbf{S}_{2}$. Indeed, the $Q_{i}$ 's may be replaced by unity because of symmetry and the remaining integrals over $\mathbf{S}_{3}$ and $\mathbf{S}_{4}$ are proportional to $\mathbf{S}_{1}$ and $\mathbf{S}_{2}$, respectively. This leads to the condition

$$
c \sum_{r}\left\{\frac{\int d \mathbf{S} S^{z} \exp \left(\beta J(r) S^{z}\right)}{\int d \mathbf{S} \exp \left(\beta J(r) S^{z}\right)}\right\}^{2}=c \sum_{r}\left\{\frac{\mathrm{I}_{\frac{m}{2}}(\beta J(r))}{\mathrm{I}_{\frac{m-2}{2}}(\beta J(r))}\right\}^{2}=1,
$$

where $\mathrm{I}_{\nu}$ is the modified Bessel function of index $\nu$. It is the generalization of the relation $c \tau_{2} \equiv c \sum_{r} \tanh ^{2} \beta J(r)=1$ for Ising spins $(m=1)$, derived in section II 6. For Heisenberg spins $(m=3)$ eq. (86) becomes

$$
c \sum_{r}\left\{\operatorname{cotanh} \beta J(r)-\frac{1}{\beta J(r)}\right\}^{2}=1 .
$$

As it involves the classical Langevin function for our classical spins, one expects it to contain to the quantum Langevin function for quantum spins. Eq. (87) is quite natural, as the same mean field form, but without taking the square, is known to occur for the ferromagnetic transition.

Eq. (87) again leads to concentration scaling $T_{G} \sim c$ for RKKY couplings. We have tested it for the situation considered by Matsubara and Iguchi. [30] These authors consider a fcc lattice with lattice constant $a_{0}$ and with $c=5 \%$ spins present. The RKKY coupling $J(r)=J_{0} \cos \left(2 k_{F} r\right)\left(a_{0} / r\right)^{3}$ involves the parameter $k_{F}=4.91 / a_{0} ; J(r)$ is set to zero for $r \geq 3 a_{0}$. The simulations yield a spin glass transition at $T_{g} / J_{0}=0.068 \pm 0.008$. We find from the above relation that $T_{g} / J_{0}=0.306$. It shows that $c=5 \%$ is not a small concentration, and that higher order corrections in $c$ are important.

\section{SPIN GLASS BEHAVIOR AT LOW TEMPERATURES}

In order to see whether spin glass behavior in the form of breaking of replica symmetry is likely to occur, we consider the simplest generalization of the SK-model. It is given by eq. (17), (18), and 19). The model represents the site-diluted spin system in a Gaussian approximation.

Consider the low temperature behavior of the replica symmetric solution $M_{\alpha}=M, q_{\alpha \alpha}=q_{d}, q_{\alpha \neq \beta}=q$. For small $T$ we observe the "groundstate dominance" $s_{\alpha}^{(j)}=s_{\alpha}^{(1)}$ for all $j=1, \cdots, \ell$. This implies that repeated spin sums reduce to single spin sums. It leads to a free energy

$$
\begin{aligned}
\beta F & =\frac{T}{2 c \hat{J}(0)} m^{2}+\frac{1}{2 c} \int \frac{\mathrm{d}^{d} k}{(2 \pi)^{d}}\left\{\ln \left(1-c \beta \hat{J}(k)\left(q_{d}-q\right)\right)-\frac{c \beta \hat{J}(k) q}{1-c \beta \hat{J}(k)\left(q_{d}-q\right)}\right\} \\
& +\frac{1}{2}\left(p_{d} q_{d}-p q\right)-\sum_{\ell} \gamma_{\ell} \int g(x) \mathrm{d} x \ln 2 \cosh \ell(h+m+x \sqrt{p}),
\end{aligned}
$$

where $g(x)=\exp \left(-x^{2} / 2\right) / \sqrt{2 \pi}$ is the Gaussian weight. It can be checked that $q_{d}=1-c$. The other mean field equations are

$$
\begin{aligned}
M & \equiv \frac{T m}{c \hat{J}(0)}=\int g(x) d x \sum_{\ell} \gamma_{\ell} \ell \tanh \ell(h+m+x \sqrt{p}), \\
q & =\int g(x) d x \sum_{\ell} \gamma_{\ell} \ell^{2} \tanh ^{2} \ell(h+m+x \sqrt{p})
\end{aligned}
$$




$$
\begin{aligned}
p & =\int \frac{\mathrm{d}^{d} k}{(2 \pi)^{d}} \frac{c \beta^{2} \hat{J}^{2}(k)}{\left(1-c \beta \hat{J}(k)\left(q_{d}-q\right)\right)^{2}}, \\
p_{d} & =p+\int \frac{\mathrm{d}^{d} k}{(2 \pi)^{d}} \frac{\beta \hat{J}(k)}{1-c \beta \hat{J}(k)\left(q_{d}-q\right)} .
\end{aligned}
$$

For small $T$ it holds that $p \sim T^{-2}$ is large. One therefore finds

$$
q=q_{d}-\sqrt{\frac{2}{\pi}} \sum_{\ell} \gamma_{\ell} \frac{\ell^{2}}{\ell \sqrt{p}}=q_{d}-\sqrt{\frac{2}{\pi p}} .
$$

Note that this result also holds for $c>1 / 2$, where the $\ell$-sum is formally divergent.

According to the analysis by de Almeida and Thouless [31], the instability of the replica-symmetric solution is caused by a negative eigenvalue of the fluctuation matrix. This mode has been termed "replicon". [32] We have proposed the name "ergodon" for it, since this name relates to the underlying physical mechanism of ergodicity breaking, rather than to one of the mathematical ways to study it. 33 The "mass" of the "ergodon" or "replicon" can be derived along the lines of AT. One expands the free energy up to second order in $\delta p_{\alpha \beta}, \delta q_{\alpha \beta}$, imposing the condition $\sum_{\alpha} \delta p_{\alpha \beta}=\sum_{\alpha} \delta q_{\alpha \beta}=0$. This finally leads to a 2 times 2 matrix, which has one large eigenvalue (not of interest to us) and an eigenvalue that may become zero and negative. The latter eigenvalue has in case of ground state dominance the approximate form

$$
\Lambda(T) \approx 1-\frac{p}{q} \sum_{\ell} \gamma_{\ell} \int g(x) \mathrm{d} x \frac{\ell^{4}}{\cosh ^{4} \ell x \sqrt{p}} .
$$

Using $q \rightarrow q_{d}=1-c$, this leads to

$$
\Lambda(T) \approx 1-(1-2 c) \sqrt{\frac{8 p}{9 \pi}} .
$$

As $p \sim T^{-2}$, it follows that $\Lambda \sim-(1-2 c) / T$, showing the expected AT instability for $0<c<1 / 2$. However, for $c>\frac{1}{2}$ the replica symmetric mode is seen to stabilize at low $T$.

To see whether the replica symmetric state has correct physics for $c>1 / 2$, we consider the zero-point entropy. We take $m=h=0$. To order $T^{0}$ the expression (88) now becomes

$$
\beta F=\frac{1}{2 c} \int \frac{\mathrm{d}^{d} k}{(2 \pi)^{d}}\left\{\ln \left(1-c \beta \hat{J}(k)\left(q_{d}-q\right)\right)-\frac{c \beta \hat{J}(k) q}{1-c \beta \hat{J}(k)\left(q_{d}-q\right)}\right\}-\sqrt{\frac{2 p}{\pi}} .
$$

It follows that the entropy has the form

$$
S=\frac{1}{2 c} \int \frac{\mathrm{d}^{d} k}{(2 \pi)^{d}}\left\{-\ln \left(1-c \beta \hat{J}(k)\left(q_{d}-q\right)\right)-\frac{c \beta \hat{J}(k)\left(q_{d}-q\right)}{1-c \beta \hat{J}(k)\left(q_{d}-q\right)}\right\}<0 .
$$

For $T \rightarrow 0$ this goes to a finite but negative value. The same behavior occurs in the replica symmetric solution of the SK model, which follows as a limiting case of the present model.

Thus replica symmetry must be broken for all $c$ at low $T$. There is a glassy phase, possibly coexisting with (anti-) ferromagnetism. This is somewhat surprising when the replica symmetric solution is stable, but similar behavior was found in a neural network. [34] The present calculation suggests that infinite order replica symmetry breaking occurs at low $T$. Due to the "ground state dominance", it could be analyzed along the lines familiar from the SK model.

\section{GINZBURG-LANDAU EXPANSION NEAR THE SPINGLASS TRANSITION}

We use a Ginzburg-Landau expansion of the free energy to investigate which kinds of glassy phases can occur in site-disordered systems. The expansion is derived using results of section II 1. Since it includes only the lowest order overlap $\left\langle\sigma_{\alpha} \sigma_{\beta}\right\rangle$, it has the same form as the Ginzburg-Landau expansion for the SK-model [2]. The difference lies in the prefactors, which now depend on the concentration of spins and on temperature. We show that these prefactors can change sign, giving rise to phase-transitions between different glassy phase. The phase diagram of the model is studied as a function of the prefactors. 
In order to obtain the Ginzburg-Landau potential, we expand the free energy eq. (17) in the off-diagonal elements of $p_{\alpha \beta}$ and $q_{\alpha \beta}$, which vanish at the transition. In the following, we redefine $q_{\alpha \alpha}=p_{\alpha \alpha}=0$ and keep track of the diagonal elements $p_{d}$ and $q_{d}$ explicitly. We obtain

$$
\begin{aligned}
\beta F_{n}= & \frac{n}{2 c(2 \pi)^{d}} \int \mathrm{d}^{d} k \ln \left(1-c \beta \hat{J}(k) q_{d}\right)-\frac{1}{2} \sum_{j=2}^{\infty} \frac{1}{j} c^{j-1} \beta^{j} J_{j} \sum_{\alpha}\left(q^{j}\right)_{\alpha \alpha} \\
& +\frac{1}{2} \sum_{\alpha \beta} q_{\alpha \beta} p_{\alpha \beta}+\frac{n}{2} q_{d} p_{d} \\
& +n \log 2+\sum_{l=1}^{\infty} \gamma_{l}\left(1-\operatorname{tr}_{s}^{(l)} \sum_{j=0}^{\infty} \frac{1}{j !}\left(\frac{1}{2} \sum_{\alpha \beta} p_{\alpha \beta} \sigma_{\alpha} \sigma_{\beta}\right)^{j} \exp \left[\frac{1}{2} p_{d} \sum_{\alpha} \sigma_{\alpha}^{2}\right]\right),
\end{aligned}
$$

where $J_{j}$ is the $j$-th moment of the effective coupling (25)

$$
J_{j}=\int \frac{\mathrm{d}^{d} k}{(2 \pi)^{d}} \hat{J}_{\mathrm{eff}}(k)^{j} .
$$

As is discussed in section II 1, this coupling includes the effects of clustering of spins which become important at higher spin densities. The saddle point equations for $p_{\alpha \beta}$ and $q_{\alpha \beta}$ are readily obtained from eq.(95).

$$
\begin{aligned}
& p_{\alpha \beta}=\sum_{j=2}^{\infty} \beta^{j} c^{j-1} J_{j}\left(q^{j-1}\right)_{\alpha \beta}, \\
& q_{\alpha \beta}=\sum_{l} \gamma_{l} \operatorname{tr}_{s}^{(l)} \sigma_{\alpha} \sigma_{\beta} \exp X^{(l)}=\left\langle\sigma_{\alpha} \sigma_{\beta}\right\rangle .
\end{aligned}
$$

Using these equations, the $p_{\alpha \beta}$ are eliminated from the free energy. We subtract the lowest order contribution, which gives the paramagnetic background, and rescale $c \beta^{2} J_{2} q_{\alpha \beta} \rightarrow q_{\alpha \beta}$. eq.(95) now reads

$$
\beta F_{n}=\frac{1}{2} \sum_{j=2}^{\infty}\left(1-\frac{1}{j}\right) \frac{T^{j} J_{j}}{J_{2}^{j}} \sum_{\alpha}\left(q^{j}\right)_{\alpha \alpha}+\tilde{\Phi}_{n}
$$

where

$$
\tilde{\Phi}_{n}=\sum_{l=1}^{\infty} \gamma_{l}\left(1-\operatorname{tr}_{s}^{(l)} \sum_{j=0}^{\infty} \frac{1}{j !}\left(\frac{1}{2} \sum_{\alpha \beta}\left(q_{\alpha \beta}+\sum_{i} T^{i} \frac{J_{2+i}}{J_{2}^{i+1}}\left(q^{i+1}\right)_{\alpha \beta}\right) \sigma_{\alpha} \sigma_{\beta}\right)^{j} \exp \left[\frac{1}{2} p_{d} \sum_{\alpha} \sigma_{\alpha}^{2}\right]\right)
$$

We expand the free energy to fourth order. $\tilde{\Phi}_{n}$ gives the following second-order contribution

$$
\frac{1}{8} \sum_{l} \gamma_{l} \operatorname{tr}_{s}^{(l)} \sum_{\alpha_{1} \beta_{1} \alpha_{2} \beta_{2}} q_{\alpha_{1} \beta_{1}} q_{\alpha_{2} \beta_{2}} \sigma_{\alpha_{1}} \sigma_{\beta_{1}} \sigma_{\alpha_{2}} \sigma_{\beta_{2}} \exp \left[\frac{1}{2} p_{d} \sum_{\alpha} \sigma_{\alpha}^{2}\right] .
$$

The trace only has a non-zero value if the replica-indices are pair-wise equal. Because $q_{\alpha \alpha}=0, \alpha_{1}=\beta_{1}$ and $\alpha_{2}=\beta_{2}$ give no contribution. Eq.(101) thus gives

$$
\frac{1}{4} \mu_{22} \sum_{\alpha}\left(q^{2}\right)_{\alpha \alpha},
$$

where we have introduced the $l$-spin moments $\mu_{k_{1} \cdots k_{m}}$

$$
\begin{aligned}
\mu_{k_{1} \cdots k_{m}} & =\sum_{l} \gamma_{l} \operatorname{tr}_{s}^{(l)} \sigma_{1}^{k_{1}} \cdots \sigma_{m}^{k_{m}} \exp \left[\frac{1}{2} p_{d} \sum_{\alpha} \sigma_{\alpha}^{2}\right] \\
& =\sum_{l} \gamma_{l} m_{k_{1}}^{(l)} \cdots m_{k_{m}}^{(l)}\left(m_{0}^{(l)}\right)^{n-m} \\
& \stackrel{n \rightarrow 0}{=} \sum_{l} \gamma_{l} \frac{m_{k 1}^{(l)}}{m_{0}^{(l)}} \cdots \frac{m_{k m}^{(l)}}{m_{0}^{(l)}}
\end{aligned}
$$


with $m_{k}^{(l)}=\operatorname{tr}_{\sigma} \sigma^{k} \exp \left[p_{d} \sigma^{2} / 2\right]$.

The third-order contribution from $j=3$ in eq.(100) is derived in the same way. The replica-indices can be paired up in eight ways, each yielding a term $\sum_{\alpha}\left(q^{3}\right)_{\alpha \alpha}$. From $j=2$ comes a term of the same form.

Both $j=2$ and $j=3$ give the fourth order term $\sum_{\alpha \alpha}\left(q^{4}\right)_{\alpha \alpha}, j=4$ gives:

$$
\begin{aligned}
& \sum_{\alpha \beta} q_{\alpha \beta}^{4} 8 \text { ways. } \\
& \left.\sum_{\alpha \beta \gamma} q_{\alpha \beta}^{2} q_{\alpha \gamma}^{2}\right|_{\beta \neq \gamma} 48 \text { ways, } \\
& \left.\sum_{\alpha \beta \gamma \delta} q_{\alpha \beta} q_{\beta \gamma} q_{\gamma \delta \delta} q_{\delta \alpha}\right|_{\alpha \neq \gamma ; \beta \neq \delta} 48 \text { ways, } \\
& \left.\sum_{\alpha \beta \gamma \delta} q_{\alpha \beta}^{2} q_{\gamma \delta}^{2}\right|_{\alpha \neq \gamma, \delta ; \beta \neq \gamma, \delta} .12 \text { ways }
\end{aligned}
$$

Since $\left(\sum_{\alpha \beta} q_{\alpha \beta}^{2}\right)^{2}$ is of order $n^{2}$, the last term in eq. (104) will not contribute in the limit $n \rightarrow 0$. We implement the constraints on the indices by Kronecker delta's and then sum them out. For the third term in eq. (104), this for instance gives

$$
\begin{aligned}
\left.\sum_{\alpha \beta \gamma \delta} q_{\alpha \beta} q_{\beta \gamma} q_{\gamma \delta} q_{\delta \alpha}\right|_{\alpha \neq \gamma ; \beta \neq \delta} & =\sum_{\alpha \beta \gamma \delta} q_{\alpha \beta} q_{\beta \gamma} q_{\gamma \delta} q_{\delta \alpha}\left(1-\delta_{\alpha \gamma}\right)\left(1-\delta_{\beta \delta}\right) \\
& =\sum_{\alpha \beta \gamma \delta} q_{\alpha \beta} q_{\beta \gamma} q_{\gamma \delta} q_{\delta \alpha}-2 \sum_{\alpha \beta \gamma} q_{\alpha \beta}^{2} q_{\alpha \gamma}^{2}+\sum_{\alpha \beta} q_{\alpha \beta}^{4} .
\end{aligned}
$$

Combining these results with the contributions from the first term in eq. (99), we finally obtain the following free energy, which has the same form as for the SK model.

$$
\beta F_{n}=-\frac{\tau}{2} \sum_{\alpha}\left(q^{2}\right)_{\alpha \alpha}-\frac{w}{6} \sum_{\alpha}\left(q^{3}\right)_{\alpha \alpha}-\frac{y_{1}}{8} \sum_{\alpha \beta} q_{\alpha \beta}^{4}-\frac{y_{2}}{8} \sum_{\alpha \beta \gamma} q_{\alpha \beta}^{2} q_{\alpha \gamma}^{2}-\frac{y_{3}}{8} \sum_{\alpha}\left(q^{4}\right)_{\alpha \alpha} .
$$

The prefactors in this expansion are functions of concentration and temperature. The prefactor of the quadratic term, $\tau=\left(\mu_{22}-T^{2} / c J_{2}\right) / 2$, vanishes at the spin glass temperature $T_{g}(c) \equiv \sqrt{c J_{2} \mu_{22}}$. The other prefactors in the free energy are given by

$$
\begin{aligned}
w & =\mu_{222}-2 \frac{T^{3} J_{3}}{c J_{2}^{3}}+3 \mu_{22} \frac{T J_{3}}{J_{2}^{2}}, \\
y_{1} & =\frac{3}{2} \mu_{2222}+\frac{1}{6} \mu_{44}-\mu_{422}, \\
y_{2} & =\mu_{422}-3 \mu_{2222}, \\
y_{3} & =\mu_{2222}-3 \frac{T^{4} J_{4}}{c J_{2}^{4}}+2 \mu_{22} \frac{T^{2}\left(J_{3}^{2}+2 J_{2} J_{4}\right)}{J_{2}^{4}}+4 \mu_{222} \frac{T J_{3}}{J_{2}^{2}} .
\end{aligned}
$$

The paramagnetic behavior is coded in the parameters $p_{d}$ and $q_{d}$, that satisfy the coupled mean field equations $p_{d}=\beta J_{1}$ and $q_{d}=\mu_{2}$. All information on clustering is contained in $\tau, w$, and the $y$ 's, so in the $\mu$ 's and the $J_{j}$. In the limit $c \rightarrow 0$ the $\mu$ 's go to unity and for $T \sim \sqrt{c}$ the $J_{3}$ and $J_{4}$ terms vanish, so that one recovers the Ginzburg-Landau free energy of the SK-model. The important factors then are $w=1, y_{1}=2 / 3$, while the values of $y_{2}(=-2)$ and $y_{3}$ $(=1)$ are irrelevant. When following the transition line $T=T_{g}(c)$ in the $c-T$ phase diagram as function of $c$, it is seen that the higher $\mu$ 's are rapidly oscillating functions. For instance, if $J_{3}$ and $J_{4}$ are much smaller than $J_{2}$, then $y_{1}$ changes sign at $c=2.7 \%$ and at $c=4.3 \%$, while $w$ becomes negative at $6.7 \%$.

Based on these observations we are led to assume that the relevant physics near the phase transition(s) is still contained in the GL free energy eq. (106). However, there is no reason to assume that $w$ and $y_{1}$ will always be positive. (A sign change of $y_{1}$ occurs also in a Potts glass. [35]) Given the type of the lattice and the values of the spin-spin couplings, the $c-T$ phase diagram may exhibit a limited number of special points $\left(c_{*}, T_{*}\right)$ where either $w$ or $y_{1}$ vanishes, and new phase boundaries originate. In fig. 1, a fictitious phase diagram is depicted for a system where $w$ changes sign at some $\left(c_{*}, T_{*}\right)$.

In the limit $n \rightarrow 0$, the free energy can be expressed in terms of the Parisi order parameter function $q(x)$ in the usual way [6]. 


$$
\beta F=\int_{0}^{1} \mathrm{~d} x\left\{\frac{\tau}{2} q^{2}(x)-\frac{w}{3} q(x) T(x)+\frac{y_{1}}{8} q^{4}(x)-\frac{y_{2}+y_{3}}{8} q^{2}(x) \int_{0}^{1} \mathrm{~d} y q^{2}(y)+\frac{y_{3}}{2} T^{2}(x)\right\},
$$

where

$$
T(x)=\frac{1}{2} x q^{2}(x)+q(x) \int_{x}^{1} \mathrm{~d} y q(y)+\frac{1}{2} \int_{0}^{x} \mathrm{~d} y q^{2}(y) .
$$

The phasediagram of this model is determined by the parameters $w$ and $y_{1}$. The sign of the cubic prefactor determines whether the model exhibits a second or a first order transition. For positive $y_{1}$, the low temperature phase will be replica symmetry broken, for negative $y_{1}$ replica symmetric. In the region where $y_{1}$ is of order $\tau^{2}$, a sixth order replica symmetry breaking term becomes relevant. We will discuss this case in section VIIB. The parameters $y_{2}$ and $y_{3}$ are only important in the region where $w \ll 1$, which we will discuss first.

\section{A. Behavior near first order type phase transitions}

Suppose we take $w$ negative and $y_{1}$ positive. As we lower temperature, the system undergoes a first order transition to a state which breaks replica symmetry. At positive $w$, on the other hand, we get a second order transition and the low temperature state will undoubtedly be different from the one at negative $w$ (although it will also break replica symmetry, since we keep $y_{1}$ positive). We want to study the transition between these two low-temperature states. We therefore take $y_{1}>0$ and allow $w$ to change sign. For $w \sim \sqrt{\tau} \ll 1, q$ is also proportional to $\sqrt{\tau}$, so eq. (111) then includes all terms of order $\tau^{2}$ and should describe the behavior near this transition.

At positive $w$, we find the usual transition from the paramagnet (PM) to a Parisi-type spin glass phase (SG) with infinite replica symmetry breaking. At $w<0$, however, the order parameter in the low-temperature phase only has one-step replica symmetry breaking (1RSB). Solutions of this form have previously been found by Gross, Kanter and Sompolinsky [35] for the Potts glass and by Crisanti, Horner and Sommers (CHS) [36] for a spherical spin glass model with $p$-spin interaction. We find a continuous transition between the SG and the 1RSB phase (fig. 3).

The prefactors $y_{2}$ and $y_{3}$, which are only important if $w$ is small, must obey the following conditions: $y_{3}<-y_{1}$ and $y_{2}>\max \left[0,-2 y_{1}-y_{3}\right]$. The first is related to keeping the plateau $q_{1}$ positive, the second to doing this for the breakpoint $x_{1}\left(q_{1}\right.$ and $x_{1}$ are defined below).

\section{The spin glass (SG I) solution}

First, we need to derive Parisi's infinite-RSB solution in the presence of the $y_{2}$ and $y_{3}$-term. We use the following ansatz for the order parameter function $q(x)$ :

$$
q(x)= \begin{cases}q_{n c}(x) & ; \quad x_{0} \leq x \leq x_{1} \\ q_{1} \equiv q_{n c}\left(x_{1}\right) & ; \quad x_{1} \leq x \leq 1\end{cases}
$$

Because $q(x)$ is continuous, it is completely determined by the saddle-point equation $\delta F / \delta q(x)=0$,

$$
\begin{aligned}
& \tau q(x)-w T(x)+\frac{y_{1}}{2} q^{3}(x)-\frac{y_{2}+y_{3}}{2} q(x) \int_{0}^{1} \mathrm{~d} y q^{2}(y) \\
& +y_{3}\left[T(x) R(x)+\int_{0}^{x} \mathrm{~d} y T(y) q(y)+q(x) \int_{x}^{1} \mathrm{~d} y T(y)\right]=0,
\end{aligned}
$$

where

$$
R(x)=x q(x)+\int_{x}^{1} \mathrm{~d} y q(y) .
$$

Both $T(x)$ and $R(x)$ are constant on the plateau at $x \geq x_{1}$.

The stability of the SG solution is determined by the sign of the "ergodon"-function $\Lambda(x)$, which is an eigenvalue of the fluctuation-matrix $\delta^{2} F[q] / \delta q(x) \delta q(y)$.

$$
\Lambda(x)=\tau-w R(x)+\frac{3 y_{1}}{2} q^{2}(x)-\frac{y_{2}+y_{3}}{2} \int_{0}^{1} \mathrm{~d} y q^{2}(y)+y_{3}\left[R^{2}(x)+x T(x)+\int_{x}^{1} \mathrm{~d} y T(y)\right] .
$$


Differentiating the saddle-point equation eq. (114) with respect to $x$, we obtain $q^{\prime}(x) \Lambda(x)=0$. This implies that the SG solution is marginally stable on the interval $0 \leq x \leq x_{1}$. Moreover, it is also marginally stable for $x>x_{1}$, because $q(x)$ is continuous at the breakpoint. This is of course a well-known feature of this state.

The fourth derivative of the saddle-point equation with respect to $x$ yields a second order differential equation for $q_{n c}(x)$. Its second derivative can be used to fix the height of the plateau $q_{1}$. We obtain,

$$
q_{n c}(x)=\frac{w \sqrt{y_{1}+y_{3} x_{1}^{2}} x}{3\left(y_{1}+y_{3} x_{1}\right) \sqrt{y_{1}+y_{3} x^{2}}} .
$$

Finally, the breakpoint $x_{1}$ is given by the implicit equation $\Lambda\left(x_{1}\right)=0$.

$$
\sigma=\frac{x_{1}}{3\left(y_{1}+y_{3} x_{1}\right)}-\frac{\left(3 y_{1}+3 y_{3}-2 y_{2}\right) x_{1}^{2}+\frac{y_{2}}{y_{3}}\left(y_{1}+y_{3} x_{1}^{2}\right)\left(x_{1}-\alpha \operatorname{atanh}\left(\frac{x_{1}}{\alpha}\right)\right)}{18\left(y_{1}+y_{3} x_{1}\right)^{2}}
$$

where $\sigma=\tau / w^{2}$ and $\alpha=\sqrt{-y_{1} / y_{3}}$. Near the transition, this yields $x_{1} \simeq 3 y_{1} \sigma$.

For $\sigma \ll 1, w>0$, eq.(117) reproduces Parisi's solution of the SK model with $x_{1} \ll 1$ (fig. 2a). When $x_{1}$ approaches $\alpha, q(x)$ squeezes into a stepfunction, with the discontinuity located at $x=x_{1}$ (fig.2f $\mathrm{f}$ ). This is the transition from infinite to one-step replica symmetry breaking. It occurs in the region $w<0$, at $\sigma=\sigma_{c}=1 / 6 y_{3}+y_{2} / 18 y_{3}^{2}(1-\alpha)$ (fig. 3).

\section{One-step replica symmetry breaking solutions}

The order parameter in the 1RSB state has the form of a step function

$$
q(x)= \begin{cases}0 & ; 0 \leq x \leq x_{1} \\ q_{1} & ; \quad x_{1} \leq x \leq 1\end{cases}
$$

Two conditions are needed in order to fix both the plateau $q_{1}$ and the breakpoint $x_{1}$. Next to the saddle-point equation $\partial F / \partial q_{1}=0$, we may impose either stationarity with respect to the breakpoint, $\partial F / \partial x_{1}=0$, or marginal stability of the ergodon on one of the plateaus, $\Lambda_{0 / 1}=0$. We will consider both procedures. The first gives the proper static solution (or the solution for an infinitely slow cooling rate), while the second solution ought to occur in dynamics.

The saddle-point equation $\partial F / \partial q_{1}=0$ reads

$$
\tau-w\left(1-\frac{1}{2} x_{1}\right) q_{1}+\frac{1}{2}\left(y_{1}-y_{2}\left(1-x_{1}\right)+3 y_{3}\left(1-x_{1}\right)+y_{3} x_{1}^{2}\right) q_{1}^{2}=0 .
$$

The static solution must satisfy the additional equation

$$
-\tau+w\left(1-\frac{2}{3} x_{1}\right) q_{1}-\frac{1}{4} y_{1} q_{1}^{2}+\frac{1}{2} y_{2}\left(1-x_{1}\right) q_{1}^{2}-y_{3}\left(\frac{3}{2}-x_{1}+\frac{3}{4} x_{1}^{2}\right) q_{1}^{2}=0 .
$$

The breakpoint of the marginally stable solution is fixed by

$$
\Lambda_{1}=0 \Leftrightarrow-\tau+w q_{1}-\frac{3}{2}\left(y_{1}+y_{3}\right) q_{1}^{2}+\frac{1}{2} y_{2}\left(1-x_{1}\right) q_{1}^{2}=0
$$

In principle, the condition $\Lambda_{0}=0$ could also yield a dynamical solution, but this 1 RSB state turns out to be unstable.

We find the following static solution

$$
q_{1}^{\text {stat }}=\frac{w x_{1}}{\frac{3}{2} y_{1}+3 y_{3} x_{1}\left(1-\frac{1}{2} x_{1}\right)}
$$

This solution sets in from $x_{1}=1$, at a negative value of $\sigma, \sigma_{1}^{\text {stat }}=1 / 9\left(y_{1}+y_{3}\right)$. The transition is first order in the sense that the order-parameter changes discontinuously and that the transition occurs before the AT instability of the PM phase. There is no latent heat, however. In fact, there is a problem, because the continuation of the PM phase has a lower free energy than the new 1RSB solution. The system nonetheless needs to go to the 1 RSB state, because the PM becomes unstable at $\sigma=0$. This problem was also encountered by CHS.

The ergodon mass of the static $1 \mathrm{RSB}$ is the same on both plateaus 


$$
\Lambda_{0}^{\text {stat }}=\Lambda_{1}^{\text {stat }}=-\frac{1}{4}\left(y_{1}+y_{3} x_{1}^{2}\right)\left(q_{1}^{\text {stat }}\right)^{2} .
$$

It goes unstable at the transition to the SG phase, $\sigma=\sigma_{c}$, where $x_{1}$ becomes equal to $\alpha$.

The dynamic solution is given by

$$
q_{1}^{\operatorname{marg}}=\frac{w x_{1}}{2 y_{1}+y_{3} x_{1}\left(3-x_{1}\right)} .
$$

It also sets in a $x_{1}=1$, but at a higher temperature than the static 1 RSB: $\sigma_{1}^{\operatorname{marg}}=1 / 8\left(y_{1}+y_{3}\right)$. Close to $\sigma_{1}^{\operatorname{marg}}$, its free energy is lower than that of the PM ( $\Delta F$ even has a finite slope, but there is no latent heat [37], [38]). However, it becomes higher than $F_{\mathrm{PM}}$ before the PM goes unstable at $\sigma=0$, so we have the same problem as with the static solution.

The dynamic solution has $\Lambda_{1}=0$, while the other eigenvalue is given by

$$
\Lambda_{0}^{\mathrm{marg}}=-\frac{1}{2}\left(y_{1}+y_{3} x_{1}^{2}\right)\left(q_{1}^{\mathrm{marg}}\right)^{2} .
$$

It vanishes at the transition to the SG phase.

The subject of dynamical transitions has made large progress since the start of this work, for a review see [39]. It is now understood that the marginality criterion signals dynamics in the highest, marginal states. This is the regime reached when first taking the thermodynamic limit and then letting time become large. In realistic systems one expects to go to lower states in the course of time, in a way that can be fixed, to some extent, by the cooling procedure. That happens in mean field spin glasses provided one considers dynamics at time scales exponential in the system size [40]. In other words, the marginality only points at the onset of a more interesting behavior. Dynamics in marginal states itself has a very limited meaning; it is a mean field artefact. The final result of these investigations has been the development of a picture for the thermodynamics of the non-equilibrium glassy state. Apart from the real temperature there occurs an effective temperature $T_{e}=T / x_{1}$, at which the system's slow modes are at quasi-equilibrium [40] [41]. This approach explains the old paradoxes related to the Ehrenfest relations and the Prigogine-Defay ratio [38], that were the basis for the general belief that thermodynamics does not work for the glassy state.

\section{B. The transition from the Edwards-Anderson to the spin glass phase}

Another region of interest is where the prefactor of the replica symmetry breaking term, $y_{1}$, changes sign. For $y_{1} \sim-1, w \sim 1$, the model is in the Edwards Anderson (EA) phase, which has a replica symmetric order parameter (fig.2e). At positive $y_{1}$, it is in the SG phase. At the transition between these two phases, $y_{1} \ll 1$ and higher order replica symmetry breaking terms become relevant. We therefore consider the following free energy

$$
\beta F_{n}=-\frac{\tau}{2} \sum_{\alpha}\left(q^{2}\right)_{\alpha \alpha}-\frac{w}{6} \sum_{\alpha}\left(q^{3}\right)_{\alpha \alpha}-\frac{y_{1}}{8} \sum_{\alpha \beta} q_{\alpha \beta}^{4}-\frac{y_{5}}{8} \sum_{\alpha \beta} q_{\alpha \beta}^{3}\left(q^{2}\right)_{\alpha \beta}-\frac{y_{6}}{6} \sum_{\alpha \beta} q_{\alpha \beta}^{6} .
$$

The $y_{2}$ and $y_{3}$ terms are omitted, since they are not important for $w \sim 1$. We have included the most dangerous fifth and sixth order term. The full list of fifth order terms is

$$
\begin{aligned}
& -\sum_{\alpha}\left(q^{5}\right)_{\alpha \alpha}\left(\frac{1}{10} \mu_{22222}+\frac{1}{2} \mu_{22} T^{3} \frac{J_{5} J_{2}+J_{3} J_{4}}{J_{2}^{5}}+\frac{1}{2} \mu_{222} T^{2} \frac{J_{4} J_{2}+J_{3}^{2}}{J_{2}^{4}}+\frac{1}{2} \mu_{2222} T \frac{J_{3}}{J_{2}^{2}}-\frac{2}{5} \frac{T^{5} J_{5}}{c J_{2}^{5}}\right) \\
& -\sum_{\alpha \beta} q_{\alpha \beta}^{3}\left(q^{2}\right)_{\alpha \beta}\left(\frac{3}{4} \mu_{22222}-\frac{1}{2} \mu_{4222}+\frac{1}{12} \mu_{442}+\frac{1}{2} T \frac{J_{3}}{J_{2}^{2}} y_{1}\right) \\
& -\sum_{\alpha \beta} q_{\alpha \beta}^{2}\left(q^{3}\right)_{\alpha \alpha}\left(-\frac{3}{4} \mu_{22222}+\frac{1}{4} \mu_{4222}\right)-\sum_{\alpha \beta \gamma} q_{\alpha \beta}^{2}\left(q^{3}\right)_{\gamma \gamma} \frac{1}{24} \mu_{22222}
\end{aligned}
$$

The last of those is of order $n^{2}$ and does not contribute for $n \rightarrow 0$. The first and the third term are also neglected, since they have a structure similar to the cubic term, but are of higher order in $\tau$. It turns out that the $y_{5}$-term (the second of eq. (128) ) can be absorbed into a redefinition of $y_{1}$ by using the saddle point equation $\left(q^{2}\right)_{\alpha \beta} \simeq-2 \tau q_{\alpha \beta} / w$. We therefore replace $y_{1}$ by $\tilde{y}_{1}=y_{1}-2 \tau y_{5} / w$ and omit the fifth order term. The prefactor of the most dangerous sixth order term is given by 


$$
y_{6}=-\frac{15}{4} \mu_{222222}+\frac{15}{4} \mu_{42222}-\frac{1}{4} \mu_{6222}-\frac{15}{16} \mu_{4422}+\frac{1}{8} \mu_{642}-\frac{1}{240} \mu_{66} \text {. }
$$

We may expect new behavior when $\tilde{y}_{1}$ and $y_{6}$ become of the same order of magnitude. Writing $\tilde{y}_{1}=2 y_{4} \tau^{2} / w^{2}$, we study the phasediagram as a function of $y_{4}$ for $\tau \ll 1$ and fixed. We find the following results:

For $y_{6}>0$, the model has a SG phase with nonzero lower plateau $q_{0}$. It interpolates between the EA phase, where $q_{0}=q_{1}$, and the SG phase, where $q_{0}=0$ (fig.4).

For negative $y_{6}$, there are two saddle points at the transition between the EA and the SG phase. The metastable saddle point consists of a 1RSB solution which sets in from the EA phase, followed by a transition to a new phase which we call SGIV (fig.2d). At larger $y_{4}$ there is a second transition from SGIV to SG.

The order parameter of the stable saddle point is shown in fig.21. This is also a new solution, which we call SGIII. It has a lower free energy than the $1 \mathrm{RSB}$ and the SGIV solution. The SGIII sets in from the EA phase with $x_{1}=q_{0}=0$, and evolves into the SG at higher $y_{4}$, where $q_{0}$ becomes equal to $q_{1}$.

The phasediagram for this region of parameter space is shown in fig.5.

The solutions in this region are obtained in much the same way as the SG and the $1 \mathrm{RSB}$. We therefore only show that there is no direct transition from the EA to the SG phase and then simply state the results for the phases which occur in between.

As $n \rightarrow 0$, the free energy eq. (127) takes the following form in terms of the order parameter function $q(x)$

$$
\beta F=\int_{0}^{1} \mathrm{~d} x\left\{\frac{\tau}{2} q^{2}(x)-\frac{w}{3} q(x) T(x)+\frac{y_{4} \tau^{2}}{4 w^{2}} q^{4}(x)-\frac{y_{6}}{6} q^{6}(x)\right\} .
$$

Taking a replica symmetric ansatz, we now find for the order parameter in the EA phase

$$
q_{\mathrm{EA}}=\frac{\tau}{w}+\frac{y_{4}-y_{6}}{w^{5}} \tau^{4}+\mathcal{O}\left(\tau^{5}\right)
$$

The SG solution is again determined by the saddle point equation $\delta F / \delta q(x)=0$. From the second derivative of this equation, the non-constant part of $q(x)$ is obtained

$$
x(q)=\frac{1}{w}\left(6 y_{4} q \frac{\tau^{2}}{w^{2}}-20 y_{6} q^{3}\right) .
$$

The upper plateau and the breakpoint of the SG order parameter are given by

$$
\begin{aligned}
& q_{1}=\frac{\tau}{w}+\frac{3 y_{4}-5 y_{6}}{w^{5}} \tau^{4}+\mathcal{O}\left(\tau^{5}\right), \\
& x_{1}=x\left(q_{1}\right)=\frac{6 y_{4}-20 y_{6}}{w^{4}} \tau^{3}+\mathcal{O}\left(\tau^{4}\right) .
\end{aligned}
$$

From these equations, it is seen that there is no smooth transition from the EA to the SG phase for nonzero $y_{6}$. The details of the various solutions which occur in the region $\left|y_{4}\right| \sim\left|y_{6}\right|$ are listed below. For all solution of the SG-type, the non-constant part of the order-parameter is given by eq.(132).

$\underline{\text { SG with } q_{0} \neq 0}$

$$
\begin{aligned}
& q_{0}=\frac{\tau}{w} \sqrt{\frac{y_{4}}{2 y_{6}}}+\mathcal{O}\left(\tau^{2}\right), \\
& q_{1}=q_{1 S G} ; x_{1}=x_{1 S G} .
\end{aligned}
$$

$\underline{\mathrm{SG} I I I}$

$$
\begin{aligned}
& q_{0}=-\frac{\tau}{4 w}+\frac{\tau}{4 w} \sqrt{1+\frac{8\left(y_{4}-y_{6}\right)}{3 y_{6}}}+\mathcal{O}\left(\tau^{2}\right) \\
& q_{1}=\frac{1}{w} \tau+\frac{y_{4}}{w^{4}}\left(6 q_{0} \tau^{3}-3 w q_{0}^{2} \tau^{2}\right)-\frac{y_{6}}{w^{2}}\left(20 q_{0}^{3} \tau-15 w q_{0}^{4}\right)+\mathcal{O}\left(\tau^{5}\right)
\end{aligned}
$$

$\underline{1 \mathrm{RSB}}$

$$
\begin{aligned}
q_{1} & =\frac{\tau}{w}+\frac{5 y_{4}-6 y_{6}}{2 w^{5}} \tau^{4}+\mathcal{O}\left(\tau^{5}\right), \\
x_{1} & =\frac{3 y_{4}-4 y_{6}}{w^{4}} \tau^{3} .
\end{aligned}
$$




$$
\begin{aligned}
& q_{0}=-\frac{\tau}{2 w}+\frac{\tau}{2 w} \sqrt{1+\frac{y_{4}-2 y_{6}}{y_{6}}}+\mathcal{O}\left(\tau^{2}\right) \\
& q_{1}=\frac{\tau}{w}+2 \frac{y_{4}-y_{6}}{w^{5}}\left(\tau^{4}+w q_{0} \tau^{3}\right)-\frac{y_{4}+2 y_{6}}{w^{3}} q_{0}^{2} \tau^{2}-\frac{y_{6}}{w^{2}}\left(2 q_{0}^{3} \tau-3 w q_{0}^{4}\right)+\mathcal{O}\left(\tau^{5}\right) \\
& x_{1}=2 \frac{y_{4}-y_{6}}{w^{4}}\left(\tau^{3}+2 w q_{0} \tau^{2}\right)-\frac{y_{6}}{w^{2}}\left(6 q_{0}^{2} \tau+8 w q_{0}^{3}\right)+\mathcal{O}\left(\tau^{4}\right)
\end{aligned}
$$

\section{Unsuccessful attempts}

Since the procedure for finding saddle points of the free energy is to guess an Ansatz and then to check if it works, we cannot guarantee that we have found everything there is. In particular, we have not found satisfactory solutions for the region $w<0$ and $y_{1}<0$. Also, there may be extra solutions in the regions where we have already found stable saddle points, as the results for $y_{1} \ll 1$ show. There are however some states which we definitely know not to be there, since we have unsuccessfully tried them. They are shown in fig. 6. Most notably, we have not found a stable 2RSB state (fig. 6a) for any values of the parameters. One-step and infinite replica symmetry breaking seem to be the only types of RSB that occur. Also 1RSB states with a non-zero lower plateau (in zero magnetic field) have been tried without success. States $b, c$ and $d$ were thought to evolve from the 1RSB at small $w$, but none of them do. State $b$ (SGII) does occur in the Potts glass [35].

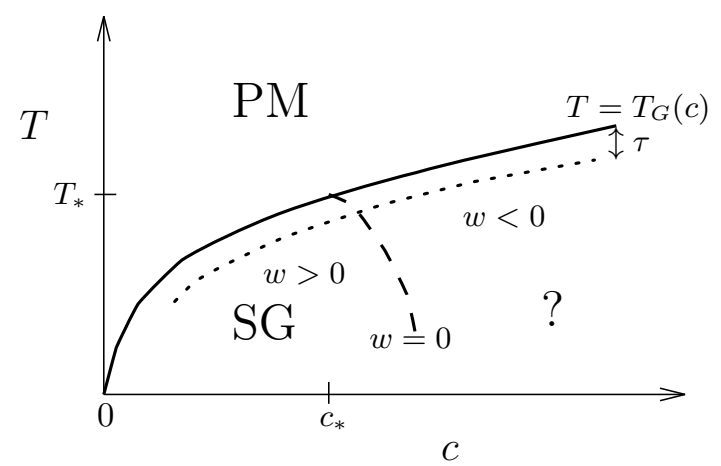

FIG. 1. $\quad c-T$ phase diagram for a fictitious system with a line $w(c, T)=0 . \mathrm{PM}=$ paramagnet; $\mathrm{SG}=$ spin glass. 
a)

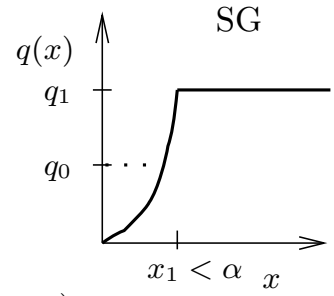

c)

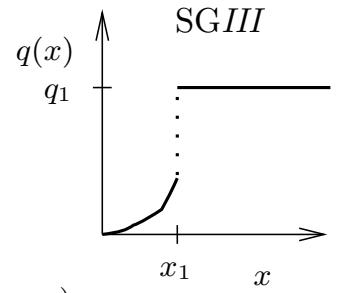

e)

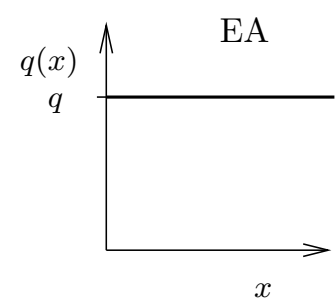

b)

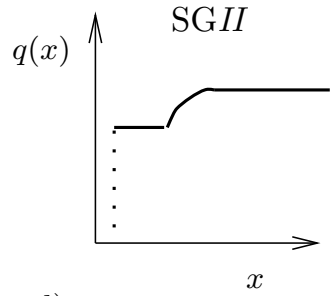

d)

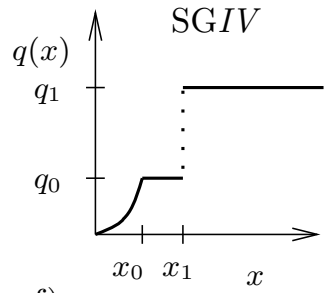

f)

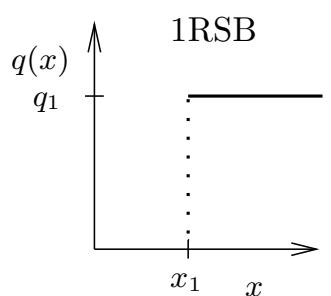

FIG. 2. The order-parameter function $q(x)$ for various phases. The SGII solution has been found for the Potts glass by Gross em et. al., but does not occur here.

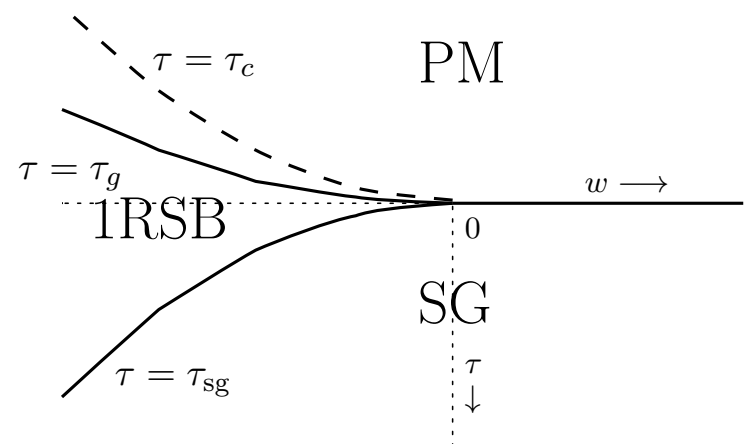

FIG. 3. $\tau-w$ phase diagram for a system with $y_{1}>0, y_{3}<-y_{1}$ and $y_{2}>\max \left[0,-2 y_{1}-y_{3}\right]$; with $w$ increasing from right to left it may appear in Fig. 1 around the point $\left(c_{*}, T_{*}\right)$. 


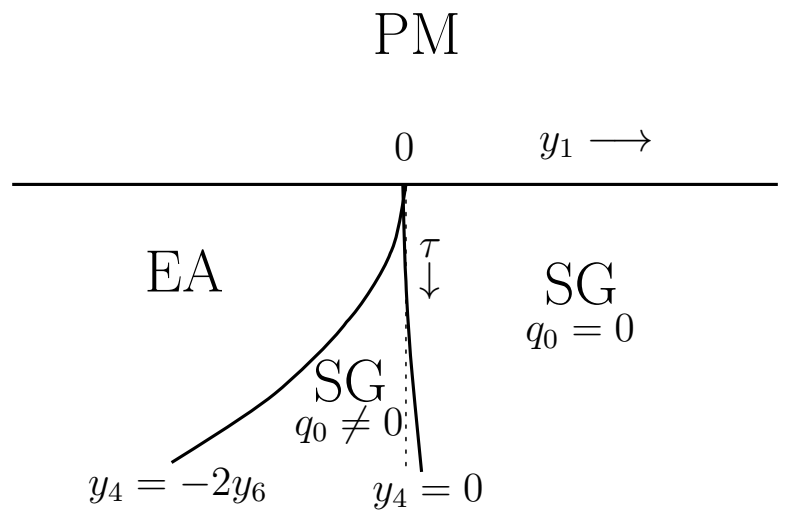

FIG. 4. $y_{1}-\tau$ phase diagram for $w>0, y_{6}>0$. The function $q(x)$ in the SG phase is drawn in figure 2 .a for the case $q_{0}=0$. In the EA-phase $q(x)$ is constant (no RSB).

\section{$\mathrm{PM}$}

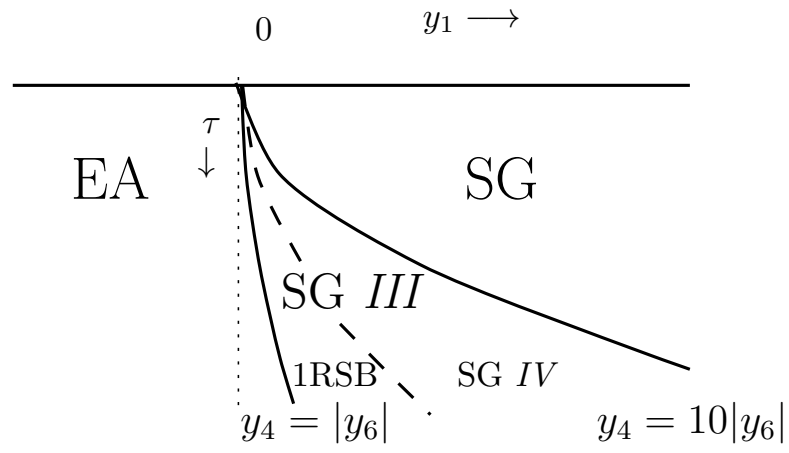

FIG. 5. $y_{1}-\tau$ phase diagram for $w>0, y_{6}<0$. In the SGIII phase $q(x)$ is as in figure 2c. Dynamically this phase splits up in a 1 RSB phase and a SG IV phase, see fig. $2 \mathrm{~b}, \mathrm{~d}$.
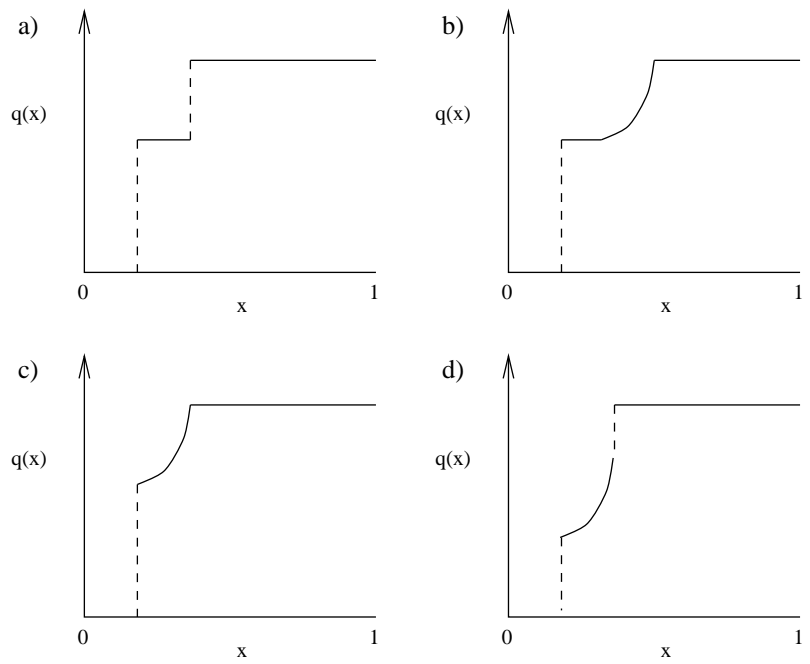

FIG. 6. Some unsuccessful attempts 


\section{SUMMARY}

We have presented the details of a new description for phase transitions in site-disordered magnets, a field that was started by us in 1992. As described in a letter of 1993 [10, the approach starts from considering an infinity of macroscopic order parameters. This approach is different from, but probably equivalent to, the space-dependent order parameter field showing up usually, such as in the model of section IV.

For the case of a random-site Ising spin glass, the central result is given by eqs. (51), (37), (18), and (49). Simplifications are found in the combined limit of small concentrations and large coordination number $z$. Here the random site problem generalizes the mean field model with random bonds, the spin glass phase of the Hopfield model and of the SK model. Only the pair overlap order parameter $q_{\alpha \beta}$ plays a role. In general, an infinity of multistate overlaps $q_{\alpha_{1} \cdots \alpha_{k}}^{(\ell)}$, and conjugated overlaps $p_{\alpha_{1} \cdots \alpha_{k}}^{(\ell)}$ occurs. A resummation has been done, which is useful for low temperatures. This approach simplifies a bit for small concentrations, where a close relation is found to the spin glass model with diluted random bonds of Viana and Bray. In this regime the random site model exhibits a percolation threshold for short range systems and the "concentration scaling" $T_{g} \sim c$ for RKKY systems.

We have generalized the approach to the case of vector spins. This has led to a criterion for the transition temperature at low concentrations.

In dilute YGd single crystals, closely related to dilute AuFe and CuMn crystals, a thermodynamic transition to a state with "complete" spin density wave formation occurs [42. The formation of incomplete spin density waves in metallic spin glasses have been observed by neutron scattering [12]. Therefore incomplete spin density waves have been presumed to be the cause of spinglass behavior in metals 43]. In our theory we indeed see that the effective coupling $J_{\text {eff }}(k)=\hat{J}(k) /\left(1-c \beta \hat{J}(k)\left(q_{d}-q_{E A}\right)\right)$ enhances the quantitative contribution of maxima in $\hat{J}(k)$. Nevertheless, thermodynamics involves all wavevectors (see, e.g. (23)) without decisive role for the special wavevectors connected to spin density waves. As expected already by many, and confirmed by the experiments of Weissman [9], incomplete spin density waves do not play a distinctive role in the spin glass phase. Although our present theory should be capable to explain why dilute YGd has spin density waves, while AuFe and CuMn have a spin glass phase, it is not clear to us how this should be shown. Let us recall that in the second order cumulant expansion (Gaussian approximation) the spin glass transition always precedes spin density waves or ferromagnetism [10.

Our present theoretical analysis also applies to the ill understood cluster glass or mictomagnetic phase. In metallic systems this is the phase observed between the low concentration spinglass and high concentration (anti-) ferromagnet, see [1] for a review. We have considered the Ginzburg-Landau expansion for our site-disordered field theory, within the second order cumulant expansion (Gaussian approximation in replica space). These expressions show that the prefactors of the cubic and quartic terms in the Ginzburg-Landau expansion may go through zero at certain points. The physically interesting cases are when either the prefactor of the cubic term vanishes, or the one of the quartic term that is responsible for replica symmetry breaking. We show that in the first case there is a transition to a phase with one step of replica symmetry breaking, while in the latter there may occur new spin glass phases between the standard spin glass and the replica symmetric Edwards-Anderson phase.

It would be interesting to analyze the clusterglass experimentally in more detail, and in particular to look for new phases. Theoretically it is worth to analyze loop effects for the new phases. They may shed insight on the question whether fluctuations (de-) stabilize the new phases, though their effect may also be a mere shift of the transition point. This problem is partly related to the notorious problem of the renormalization of the AT-line. 44 Finally the question of reentrant phases should also fall within the scope of the theory presented here.

\section{ACKNOWLEDGMENTS}

Throughout the years the authors have benefited from discussions with Jean-Philippe Bouchaud, Ton Coolen, Cyrano de Dominicis, Jean-Marc Luck, John Mydosh, Giorgio Parisi, Henri Orland, David Sherrington, and many others.

[1] J. A. Mydosh, Spin glasses - an experimental study (Taylor and Francis, London, 1993)

[2] K.H Fischer and J.A. Hertz, Spin glasses (Cambridge Studies in Magnetism 1, eds. D. Edwards and D. Melville, Cambridge, 1991) 
[3] S.F. Edwards and P.W. Anderson, J. Phys. F 5 (1975) 965

[4] D. Sherrington and S. Kirkpatrick, Phys. Rev. Lett. 35 (1975) 1972

[5] L. Viana and A.J. Bray, J. Phys. C 18 (1985) 3037

[6] M. Mézard, G. Parisi, and M.A. Virasoro, Spin Glass Theory and Beyond (World Scientific, Singapore, 1987)

[7] C. de Dominicis, I. Kondor, and T. Temesvari, in Spin glasses and random fields, edited by P. Young, (World Scientific, Singapore, 1997)

[8] E. Marinari, G. Parisi, F. Ritort and J. J. Ruiz-Lorenzo, Phys. Rev. Lett. 76 (1996) 843; N. Kawashima and A.P. Young, Phys. Rev. B 53 (1996) R484

[9] G.B. Alers, N.E. Israeloff, and M.B. Weissman, Phys. Rev. b 46 (1992) 507; M.B. Weissman, N.E. Israeloff, and G.B. Alers, Jounal of Mag. Mag. Mat. 114 (1992) 87

[10] Th.M. Nieuwenhuizen, Europhys. Lett. 24 (1993) 797

[11] Th.M. Nieuwenhuizen and C.N.A. van Duin, J. Phys. A 30 (1996) L55

[12] J.W. Cable, S.A. Werner, G.P. Felcher, and N. Wakabayashi, Phys. Rev. Lett. 49 (1982) 829;

[13] J.J. Hopfield, Proc. Natl. Acad. Sci. USA 79 (1982) 2554 (also in [6]); D.J. Amit, H. Gutfreund, and H. Sompolinsky, Phys. Rev. Lett. 55 (1985) 1530 (also in 66)

[14] A.J. Bray and M.A. Moore, J. Phys. C 15 (1982) 3897

[15] J.W. Essam, in Phase Transitions and Critical Phenomena 2, C. Domb and M.S. Green eds., (Academic, New York, 1972), p 192

[16] Th.M. Nieuwenhuizen (1984), unpublished

[17] P.J. Flory, Principles of Polymer Chemistry, (Cornell Univeristy, Ithaca, 1953; chapter 9)

[18] R.M. Ziff, E.M. Hendriks, and M.H. Ernst, Phys. Rev. Lett. 49 (1982) 593

[19] A.C.C. Coolen, R.W. Penney, and D. Sherrington, Phys. Rev. B 48 (1993) 16116; R.W. Penney, A.C.C. Coolen, and D. Sherrington, J. Phys. A 26 (1993) 3681

[20] C. de Dominicis and P. Mottishaw, J. Phys. A 20 (1987) L 1267

[21] C. De Dominicis and Y.Y. Goldschmidt, J. Phys. A 22 (1989) L775

[22] J.R.L. de Almeida, C. De Dominicis and P. Mottishaw, J. Phys. A 21 (1988) L693

[23] J.D. Honeycutt and D. Thirumalai, J. Chem. Phys. 90 (1989) 4542

[24] M. Mézard and G. Parisi, J. de Physique I (Paris) (1991) 809

[25] H. Orland and T. Garel,

[26] To apply this approach to the site disordered spin glass was proposed to one of us by H. Orland and J.P. Bouchaud.

[27] D. Dean and D.L Lancaster, Phys. Rev. Lett. 77 (1996) 3037; J. Phys. A 30 (1997) 37

[28] D. Dean and D.L Lancaster (1995), private communication

[29] M. Gabay and G. Toulouse, Phys. Rev. Lett. 47 (1981) 201

[30] F. Matsubara and M. Iguchi, Phys. Rev. Lett. 68 (1992) 3781

[31] J.R.L. de Almeida and D.J. Thouless, J. Phys. A 11 (1978) 983

[32] A. Bray and M. Moore, Phys. Rev. Lett. 41 (1978) 1068

[33] Th.M. Nieuwenhuizen, Phys. Rev. Lett. 74 (1995) 4289

[34] V.S. Dotsenko and B. Tirozzi, J. Phys. A 24 (1991) 5163

[35] D.J. Gross, I. Kanter, and H. Sompolinsky, Phys. Rev. Lett. 55 (1985) 304

[36] A. Crisanti, H. Horner, and H.J. Sommers, Z. Phys. B 92 (1993) 257

[37] Th.M. Nieuwenhuizen, Phys. Rev. Lett. 74 (1995) 3463

[38] Th.M. Nieuwenhuizen, Phys. Rev. Lett. 79 (1997) 1317

[39] J.P. Bouchaud, L. F. Cugliandolo, J. Kurchan, and M. Mézard, Physica A 226 (1996) 243

[40] Th. M. Nieuwenhuizen, J. Phys. A. Lett. 31 (1998) L201

[41] Th.M. Nieuwenhuizen, Thermodynamics of the glassy state: effective temperature as an additional system parameter, preprint 1997

[42] L.E. Wenger, G.W. Hunter, J.A. Mydosh, J.A. Gotaas, and J.J. Rhyne, Phys. Rev. Lett. 56 (1986) 1090

[43] S.A. Werner, Comm. Cond. Matter Phys. 15 (1990) 55

[44] A.J. Bray and S.A. Roberts, J. Phys. C 13 (1980) 5405 